\title{
Parallel meta-transcriptome analysis reveals degradation of plant secondary metabolites by beetles and their gut symbionts
}

\author{
Jing Wei ${ }^{1}$, Xing-Ke Yang ${ }^{2}$, Kari Segraves $^{3}$, and Huai-Jun Xue \\ ${ }^{1}$ Chongqing University School of Life Sciences \\ ${ }^{2}$ Institute of Zoology, Chinese Academy of Sciences \\ ${ }^{3}$ Syracuse University \\ ${ }^{4}$ Nankai University College of Life Sciences
}

September 27, 2021

\begin{abstract}
Switching to a new host plant is a driving force for divergence and speciation in herbivorous insects. This process of incorporating a novel host plant into the diet may require a number of adaptations in the insect herbivores that allow them to consume host plant tissue that may contain toxic secondary chemicals. As a result, herbivorous insects are predicted to have evolved efficient ways to detoxify major plant defenses and increase fitness by either relying on their own genomes or by recruiting other organisms such as microbial gut symbionts. In the present study we used parallel meta-transcriptomic analyses of Altica flea beetles and their gut symbionts to explore the contributions of beetle detoxification mechanisms versus detoxification by their gut consortium. We compared the gut meta-transcriptomes of two sympatric Altica species that feed exclusively on different host plant species as well as their F1 hybrids that were fed one of the two host plant species. These comparisons revealed that gene expression patterns of Altica are dependent on both beetle species identity and diet. The community structure of gut symbionts was also dependent on the identity of the beetle species, and the gene expression patterns of the gut symbionts were significantly correlated with beetle species and plant diet. Some of the enriched genes identified in the beetles and gut symbionts are involved in the degradation of secondary metabolites produced by plants, suggesting that Altica flea beetles may use their gut microbiota to help them feed on and adapt to their host plants.
\end{abstract}

Parallel meta-transcriptome analysis reveals degradation of plant secondary metabolites by beetles and their gut symbionts

Jing Wei ${ }^{1,3,4}$, Xing-Ke Yang ${ }^{3}$, Kari A. Segraves ${ }^{5,6,}$, Huai-Jun Xue ${ }^{2,3,{ }^{*}}$

${ }^{1}$ School of Life Sciences, Chongqing University, Chongqing 400044, China

${ }^{2}$ College of Life Sciences, Nankai University, Tianjin 300071, China

${ }^{3}$ Institute of Zoology, Chinese Academy of Sciences, Beijing 100101, China

${ }^{4}$ University of Chinese Academy of Sciences, Beijing 100049, China

${ }^{5}$ Department of Biology, Syracuse University, 107 College Place, Syracuse, NY 13244, USA

${ }^{6}$ Archbold Biological Station, 123 Main Drive, Venus, FL 33960, USA

Correspondence:

Huai-Jun Xue, College of Life Sciences, Nankai University, Tianjin 300071, China

Email:xuehj@nankai.edu.cn 
Kari A. Segraves, Department of Biology, Syracuse University, 107 College Place, Syracuse, NY 13244, USA

Email: ksegrave@syr.edu

Running title: Parallel meta-transcriptome of beetle guts

\section{Abstract}

Switching to a new host plant is a driving force for divergence and speciation in herbivorous insects. This process of incorporating a novel host plant into the diet may require a number of adaptations in the insect herbivores that allow them to consume host plant tissue that may contain toxic secondary chemicals. As a result, herbivorous insects are predicted to have evolved efficient ways to detoxify major plant defenses and increase fitness by either relying on their own genomes or by recruiting other organisms such as microbial gut symbionts. In the present study we used parallel meta-transcriptomic analyses of Altica flea beetles and their gut symbionts to explore the contributions of beetle detoxification mechanisms versus detoxification by their gut consortium. We compared the gut meta-transcriptomes of two sympatric Altica species that feed exclusively on different host plant species as well as their $\mathrm{F}_{1}$ hybrids that were fed one of the two host plant species. These comparisons revealed that gene expression patterns of Altica are dependent on both beetle species identity and diet. The community structure of gut symbionts was also dependent on the identity of the beetle species, and the gene expression patterns of the gut symbionts were significantly correlated with beetle species and plant diet. Some of the enriched genes identified in the beetles and gut symbionts are involved in the degradation of secondary metabolites produced by plants, suggesting that Altica flea beetles may use their gut microbiota to help them feed on and adapt to their host plants.

\section{Keywords}

Altica ; Chrysomelidae; adaptation; gut symbiont; meta-transcriptome; secondary metabolites; host use

\section{INTRODUCTION}

Plant feeding insects encompass a diversity of life habits and are species rich (Scheffers et al., 2012). Part of this diversity can be attributed to their ability to switch to new host plant species as this process of host switching is thought to be a key first step in divergence and speciation among herbivorous insects (Stireman et al., 2005; Simon et al., 2015; Forbes et al., 2017). Using a new host plant may require a number of adaptations, particularly those that facilitate degradation of novel plant secondary chemicals (Berenbaum \& Zangerl, 1998; Després et al., 2007; Itoh et al,. 2018). These detoxification mechanisms can be inherent adaptations of the insect that allow them to degrade and/or sequester toxic chemistry encountered when feeding on a novel host plant. Furthermore, studies of the transcriptional responses by insects feeding on different host plants have revealed a diversity of differentially expressed detoxification genes, suggesting a role for plasticity in host plant adaptation (e.g., Vogel et al., 2014; Koenig et al., 2015; Simon et al., 2015; Wybouw et al., 2015; Yu et al., 2016; Zhu et al., 2016; Orsucci et al., 2018; Näsvall et al., 2021).

Although herbivorous insects have evolved a number of mechanisms for detoxifying host plant chemistry (e.g., Després et al., 2007), we also know that gut microbes can play an important role in facilitating host use and insect diversification (Hammer \& Bowers, 2015). For example, gut symbionts may contribute to nutrition, detoxify plant secondary metabolites, provide protection from parasites and pathogens, modulate immune responses, and mediate inter- and intraspecific communication (e.g., Engel \& Moran, 2013; De Fine Licht et al., 2012; Adams et al., 2013; Boone et al., 2013; Chu et al., 2013; Hansen \& Moran, 2014; Mason et al., 2014; Ceja-Navarro et al., 2015; Douglas, 2015; Welte et al., 2016; Berasategui et al., 2017; Itoh et al., 2018; Zhang et al., 2020).

Plants with a diverse array of secondary compounds pose a major selective challenge to both herbivorous insects and their gut microbiota (Vilanova et al., 2016), and so we might expect that insect diet will have an important effect on gut microbial diversity. Interestingly, we see considerable variation in gut microbial diversity that is associated with a number of factors in addition to insect diet, including environmental habitat, developmental stage of the insect, and even the identity and phylogenetic history of the host insect 
(Colman et al., 2012; Yun et al., 2014). Some of this variation is likely caused by how the insects acquire their gut symbionts. For instance, unlike some social insects that can transmit gut symbionts both vertically and horizontally through social interactions, many insects acquire their microbes from the environment each generation, thus both seasonal and spatial variation in microbiota will impact gut microbial diversity (e.g., Jones et al., 2019; Wei et al., 2020). As a result, most insects are unable to set up intimate associations with their gut symbionts and maintain a consistent microbiota (Engel \& Moran, 2013). Furthermore, composition and diversity of gut microbiota can also be severely disrupted or eliminated during larval development when the insect molts (Engel \& Moran, 2013). Together, these studies suggest that although gut microbes may be important in helping insects to feed on a toxic diet, these interactions are unlikely to be highly speciesspecific, intimate associations. Whether transient associations between insects and gut microbes can benefit herbivorous insects remains to be tested.

In particular, there are some insect groups that are expected to have stronger mutualistic associations with their gut microbial communities. Chewing herbivorous insects, for example, such as lepidopteran and sawfly larvae, beetles, and orthopterans are predicted to be particularly reliant on microbes for detoxification because these insect groups are commonly exposed to plant secondary chemicals (Hammer \& Bowers, 2015). However, several studies on lepidopterans have shown that some caterpillar species do not rely on bacterial symbioses to consume diverse diets and they sometimes lack a resident gut microbiome (Whitaker et al., 2016; Staudacher et al., 2016; Hammer et al., 2017), suggesting that this interaction may not be important for detoxification of host plant secondary chemistry. That said, functional studies on the gut microbiota of foliarfeeding insects other than lepidopteran species are limited; thus, there is a strong need to examine other insect groups to verify whether the work in lepidopterans represents a broad-scale pattern. Furthermore, few studies have simultaneously compared the insect's endogenous detoxification mechanisms versus those attributed to their gut microbiota to determine their contributions to the ability of herbivorous insects to feed on plant defense compounds (van den Bosch \& Welte, 2017; Shukla \& Beran, 2020).

A good system in which to test ideas about the role of gut microbes in plant secondary chemistry metabolism is the leaf beetle genusAltica. These beetles have diversified in part through interactions with their host plants, and some species groups occur sympatrically, allowing us to control for environmental differences in microbial diversity. Altica fragariaeNakane and A. viridicyanea (Baly) (Insecta: Coleoptera: Chrysomelidae) are sympatric, closely-related leaf beetles that feed on distinct host plants. Altica fragariae feeds on Duchesnea indica and A. viridicyanea uses Geranium nepalense as its host plant. Neither species will feed nor oviposit on one another's host plant even under no-choice conditions, whereas the $\mathrm{F}_{1}$ hybrid generation can develop by feeding on either plant species (Xue et al., 2009a,b, 2011, 2016). These Altica species offer an interesting model system to study interactions between specialist insect herbivores and their gut symbionts. Previous work based on 16S rRNA amplicon sequencing and antibiotic treatment approaches showed that spatiotemporal variation in microbial diversity contributed more to gut bacterial composition than beetle host species, but there was only weak support that gut microbes were involved in detoxification of plant secondary chemistry (Wei et al., 2020).

In this study, we used metatranscriptome analysis to assess the contributions of beetle-derived versus gut microbe-derived sources of plant secondary chemistry degradation. Metatranscriptome analysis is a powerful tool that can reveal the genes that are actively being expressed in complex microbial communities (Gifford et al., 2011; Bashiardes et al., 2016). Here we used parallel meta-transcriptomic sequencing of whole guts of $A$. fragariae, A. viridicyanea, and their $\mathrm{F}_{1}$ hybrids to assess how beetle species and diet impacted gene expression of both beetles and their gut microbes. We had three major goals: 1) reveal transcriptomic responses of Altica beetles feeding on different host plants; 2) test whether diet shapes gut microbial communities of Altica beetles; and 3) predict the contribution of gut symbionts to detoxification of plant derived secondary chemicals.

\section{MATERIALS AND METHODS}

\subsection{Insect sampling}


Approximately 20 over-wintering adults of both A. fragariae and A. viridicyanea were collected from field populations at Changping $\left(40.11^{\prime} \mathrm{N}, 116.00^{\prime} \mathrm{E}\right)$ in Beijing, China and were maintained as colonies in the laboratory. The beetle colonies were kept in a growth chamber held at $25^{\circ} \mathrm{C}$ with a 16:8 $\mathrm{h} \mathrm{L}: \mathrm{D}$ cycle. To maintain the colonies, each species was fed their normal host plant: A. fragariae was fed Duchesnea indica and $A$. viridicyanea was fedGeranium nepalense. We sourced the plant material from the same location as the original beetle collections. This material was kept in ziplock bags in a refrigerator for no more than one week before using. The colonies were used to produce a second generation of pure parental species and their $\mathrm{F}_{1}$ hybrids that were then used in subsequent experiments. Hybrids were made between $A$. viridicyaneafemales and $A$. fragariae males because there is some reproductive isolation between the species and the reciprocal cross yields few viable offspring. We used newly hatched larvae of this second generation for a meta-transcriptome analysis. We had four experimental treatments: $A$. fragariae fed their normal host plant D. indica(abbreviated as AFDi), A. viridicyanea fed their normal host plant G. nepalense (abbreviated as AVGn), $\mathrm{F}_{1}$ hybrids fed $D$. indica (abbreviated as $\mathrm{F}_{1} \mathrm{Di}$ ) and $\mathrm{F}_{1}$ hybrids fed $G$. nepalense (abbreviated as $\mathrm{F}_{1} \mathrm{Gn}$ ) (Table 1). Using these four treatment groups, we planned to make three different comparisons of the metatranscriptome results: (1) comparison of the two parental species (AFDi vs. AVGn) (2) comparison of the hybrids fed the different host plants $\left(\mathrm{F}_{1}\right.$ Di vs. $\mathrm{F}_{1} \mathrm{Gn}$ ), and (3) comparison of all beetles fed D. indica (DiT $\left.=\mathrm{AFDi}+\mathrm{F}_{1} \mathrm{Di}\right)$ versus all beetles fed $G$. nepalense $\left(\mathrm{GnT}=\mathrm{AVGn}+\mathrm{F}_{1} \mathrm{Gn}\right)$. More than 200 newly hatched larvae for each group were fed their assigned host plant throughout larval development. Upon eclosion into adults, we continued to feed the newly emerged beetles with the same host plant species as they were given as larvae. The feeding treatments ceased when adults reached sexual maturity after ten days. At this time, the beetles were sacrificed and used for RNA extraction and sequencing.

\subsection{RNA isolation and meta-transcriptome sequencing}

To dissect the gut, we clamped the head of living beetles with sterile forceps, hauled out the whole gut gently, removed the head, and preserved the gut in RNAhold (TransGen Biotech) before RNA extraction. Guts from 30 beetles were pooled per replicate library, and we used three replicate libraries per experimental treatment for a total of 90 beetles.

Total RNA was extracted from the pooled beetle gut samples using the AllPrep DNA/RNA 96 Kit (Qiagen), following to the manufacturer's protocol. We used one $\mu \mathrm{g}$ RNA per pooled sample and used the NEBNext UltraTM RNA Library Prep Kit for Illumina (NEB, USA) to prepare the sequencing libraries, following the manufacturer's protocol. During library preparation, we added unique barcodes to each library so that we could attribute sequences to specific samples. We processed the samples by first isolating the mRNA using poly-T oligo-attached magnetic beads and then fragmenting by heating the samples in NEBNext First Strand Synthesis Reaction Buffer. Random hexaprimers were used to synthesize first strand cDNA with M-MuLV Reverse Transcriptase, and second strand cDNA was generated with DNA polymerase I and RNase H. We added poly(A) tails to the 3 ' ends and then ligated on NEBNext adapters with hairpin loop structures. cDNA fragments were size selected to $240 \mathrm{bp}$ using the AMPure XP system (Beckman Coulter, Beverly, USA) and then were incubated at $37 \mathrm{C}$ for 15 minutes with USER enzyme (NEB, USA) to cleave the hairpin loop adapter. We used Phusion High-Fidelity DNA polymerase to perform PCR and then determined the library quality using the Agilent Bioanalyzer 2100. Library preparation and sequencing was done by Biomarker Technology (Beijing, China).

In order to cluster the barcoded samples, we used the cBot Cluster Generation System and the TruSeq PE Cluster Kit v4-cBot-HS (Illumina). These libraries were then sequenced on the Illumina Hiseq Xten platform to generate paired-end reads.

\subsection{Sequence processing}

We first cleaned the sequence data by removing reads that (1) contained the adapter sequence, (2) contained a poly-N sequence, and (3) reads that were of low quality. We used in-house perl scripts to accomplish this initial data processing.

\subsection{Insect data analysis}




\subsubsection{Gene functional annotation}

The default parameters of TopHat v2.0.9 (Kim et al., 2013) were used to map the clean reads of each sample to an unannotated genome assembly for $A$. viridicyanea. We then assembled the aligned reads into individual transcripts using the software Cufflinks v2.2.1 (Trapnell et al., 2012). The predicted genes were annotated by aligning them to the following databases using BLAST v2.2.31 (Altschul et al., 1990): Nr (NCBI non-redundant protein sequences); GO (Gene Ontology) (Ashburner et al., 2000); KOG/COG (Clusters of Orthologous Groups of proteins); KEGG (Kyoto Encyclopedia of Genes and Genomes) (Kanehisa et al., 2004); Pfam (Protein family) (Finn et al., 2014); Swiss-Prot (A manually annotated and reviewed protein sequence database) (Bairoch et al., 1996) and eggNOG (Non-supervised Orthologous Groups) (Powell et al., 2014).

\subsubsection{Differential expression analysis}

Pearson's Correlation Coefficients (r) were used to assess repeatability across biological replicates within each treatment. We determined whether there was differential gene expression among treatment comparisons by conducting a differential expression analysis using the R package DESeq (v1.10.1) (Anders \& Huber, 2010). The resulting P-values were adjusted using sequential Bonferroni correction to control the false discovery rate (Benjamini \& Hochberg, 1995). We considered genes that had an adjusted P-value $<0.01$ to be differentially expressed.

\subsubsection{GO and KEGG pathway enrichment analysis}

We used the differentially expressed genes (DEGs) to conduct a gene ontology (GO) enrichment analysis. This was done using the GOseq $\mathrm{R}$ packages that adjust for potential gene length bias in DEGs (Young et al., 2010). In addition, the KEGG (Kanehisa et al., 2004) database was used to test for statistical enrichment of differentially expressed genes in KEGG pathways. We used the KOBAS (Mao et al., 2005) software to do this.

\subsection{Gut microbiota data analysis}

\subsubsection{Data filter}

We used the default parameters in Bowtie 2 (Langmead \& Salzberg, 2012) to filter the reads corresponding to beetle sequences. Because the genomes for the two host plants (D. indica and G. nepalense) are not currently available, we downloaded all of the available assembled plant genomes from the RefSeq database (Pruitt et al., 2007), and then we used Kraken 2 (Wood \& Salzberg, 2014) set to the default parameters to filter the reads associated with plants.

\subsubsection{Meta-transcriptome assembly and gene predication}

We used Trinity v2.5.1 (Grabherr et al., 2011) to assemble the remaining sequences for each sample. For this analysis, we used the default parameters and then used FragGeneScan (Rho et al., 2010) to predict microbial gene coding sequences.

\subsubsection{Construction ofnon-redundant gene sets}

To eliminate redundancy in the dataset, we used cd-hit v4.6.6 (Fu et al., 2012) (http://www.bioinformatics.org/cd-hit/) to construct a non-redundant gene dataset. We set the similarity threshold to $95 \%$ and the coverage threshold was set to $90 \%$.

\subsubsection{Microbial community composition andrelative abundance analysis}

Microbial species composition and relative abundance in each sample were determined by comparing the non-redundant genes to the Nr database. The parameters for the low count and low variance filters were set to default settings, and the samples were rarefied to the minimum library size. Using this dataset, we calculated four microbial diversity estimators, including two nonparametric richness indices (abundancebased coverage estimator (ACE) and bias-corrected Chao1), and two alpha-diversity indices (Shannon's and 
Simpson's). We used nonparametric Mann-Whitney (for two groups) or Kruskal-Wallis (for four groups) tests to assess whether there were differences among treatments for each of these estimators.

We used a combination of ordination methods (Principal coordinates analysis (PCoA) and Non-MetricMultiDimensional Scaling (NMDS)), distance methods (Jaccard and Bray-Curtis) and statistical methods (Permutational multivariate analysis of variance (PerMANOVA) and Analysis of similarities (Anosim)) to test if microbial community composition differed among feeding treatment comparisons and beetles species. Twoway PerMANOVA (with 9,999 permutations) was conducted using PAST version 3.14 (Hammer et al., 2001) to test the role of beetle species identity (AF, AV and $\mathrm{F}_{1}$ hybrid) and host plant diet (D. indica and $G$. nepalense ) in structuring gut microbial communities. For this analysis, we used "beetle species" and "host plant" as main effects. DESeq2 (Love et al., 2014) was used to determine if species composition differed among treatment comparisons. A false discovery rate (FDR) [?] 0.05 was used as the threshold to determine significant differences.

\subsubsection{Analysis of functional gene composition and abundance}

The non-redundant genes were annotated by using Blastx with a maximal e-value of $1 \mathrm{e}^{-5}$. The genes were aligned to several protein databases, including Nr, GO, KEGG, eggNOG, Pfam and SwissProt. Based on the KEGG Orthology (KO) and eggNOG Orthology datasets, we combined ordination methods (Principal Component Analysis (PCA), PCoA and NMDS), distance methods (Jaccard and Bray-Curtis) and statistical methods (PerMANOVA and Anosim) to test if the functional genes differed among treatment comparisons.

We detected microbial DEGs using edgeR (v 3.14.0) ( $\log _{2}$ (fold change) [?] 4 and p [?] 0.05). We made three comparisons of beetles that were fed different host diets: AFDi vs. AVGn, $F_{1}$ Di vs. $F_{1}$ Gn and DiT vs. GnT. Differences in the abundance of non-redundant gene enrichment in annotated KEGG pathways were tested using a right-tailed Fisher's exact test.

\subsection{Analysis platforms}

All analyses were conducted on the Biomarker Biocloud Platform (http://en.biocloud.net/private-cloud) or the MicrobiomeAnalyst platform (https://www.microbiomeanalyst.ca/ ) (Dhariwal et al., 2017; Chong et al., 2020).

\section{RESULTS}

About $34.7\left(30.5^{\sim} 46.2\right)$ gigabases $(\mathrm{Gb})$ of sequence data in $2.3 \times 10^{8}$ reads were obtained from each pooled intestinal tissue sample. Across the 12 pooled samples, we obtained an average of $4.8 \times 10^{7}$ reads from beetles and an average of $5.3 \times 10^{7}$ reads obtained from microbiota (Table 2 ).

\subsection{Beetle transcriptomic analysis}

\subsubsection{Annotated genes}

In total, 9,422 genes were predicted in Altica, and of these, 8,843 genes were annotated in at least one of the databases that we used (Table 3). We identified five multigene families that were of special interest due to their association with detoxification enzymes: cytochrome P450s (P450s), carboxyl/cholinesterases (CCEs), glutathione S-transferases (GSTs), UDP-glycosyltransferases (UGTs) and ATP binding cassette transporters (ABCs). Among the annotated genes, there were 74 P450s, 71 CCEs, 24 GSTs, 27 UGTs and 94 ABCs (Table S1), which were generally consistent with the gene numbers we obtained in a genome assembly of Altica viridicyanea (101 P450s, 97 CCEs, 27 GSTs, 32 UGTs and 69 ABCs) (Xue et al., 2021).

\subsubsection{Differentially expressed genes in Altica guts}

A heatmap of Altica gut gene expression showed high repeatability among biological replicates in each treatment (Fig. 1), ranging from 0.894 to 0.988 . We detected a large number of DEGs between the beetle species and between the diet treatments. In total, there we identified 1613 DEGs when comparing AFDi and AVGn, 252 DEGs between beetles feeding on D. indica and G. nepalense, and 164 DEGs when comparing the $\mathrm{F}_{1}$ hybrids that were fed the two different host plant species. Many of these DEGs were annotated (Table 
4). Interestingly, there were a number of DEGs associated with detoxification. For the comparison of AFDi and AVGn, we observed 26 P450 genes (20 up-regulated and 6 down-regulated), 27 CCEs (14 up-regulated and 13 down-regulated), 10 GSTs (8 up-regulated and 2 down-regulated), 10 UGTs (7 up-regulated and 3 down-regulated) and $22 \mathrm{ABCs}$ (13 up-regulated and 9 down-regulated). In the comparison of beetles feeding on the two host plants, there were 6 P450s (all up-regulated), 5 CCEs (3 up-regulated and 2 down-regulated), 3 GSTs (all up-regulated), 1 UGT (up-regulated) and 6 ABCs (3 up-regulated and 3 down-regulated) were differentially expressed. When we compared the two treatment groups for the $\mathrm{F}_{1}$ hybrids, we identified 7 P450s (all up-regulated), 4 CCEs (one up-regulated and 3 down-regulated) and 3 ABCs (one up-regulated and 2 down-regulated) that were differentially expressed (Table S2).

\subsubsection{GO and KEGG enrichment analysis}

Among the 5,009 genes annotated using the GO database, we found a number of DEGs in our comparisons. When we compared the two beetle species, we found 744 DEGs, the comparison of beetles feeding on the two plant species identified 102 DEGs, and the comparison of the F1 hybrids showed 65 DEGs (Table 4; Fig. S1). The enriched GO terms are mainly associated with catalytic activity, metabolic processes, cellular processes, single-organism processes, binding, cell part and localization (Fig. S1). In the parallel analysis based on the KEGG database, we found similar numbers of DEGs as the GO enrichment analysis (AFDi vs. AVGn: 686, DiT vs. GnT: 94, and $\mathrm{F}_{1}$ Di vs. $\mathrm{F}_{1} \mathrm{Gn}$ : 66 genes) (Fig. S2, Table 4). A comparison of DEGs between the two beetle species revealed four significantly enriched pathways that were involved in (1) glutathione metabolism, (2) cysteine and methionine metabolism, (3) glycine, serine and threonine metabolism, and (4) lysosomes. When we compared beetles feeding on the two plant species, there were four significantly enriched pathways that were involved in (1) glutathione metabolism, (2) cyanoamino acid metabolism, (3) taurine and hypotaurine metabolism, and (4) arachidonic acid metabolism (Table 5). Finally, the comparison of the F1 hybrids feeding on the two host plants identified three enriched pathways: (1) Arachidonic acid metabolism, (2) Cyanoamino acid metabolism, and (3) Taurine and hypotaurine metabolism. Of the enriched pathways that we identified, the ones that are mostly likely involved in host plant tissue metabolism are the enrichment pathways involved in amino acid metabolism, lysosome and glutathione metabolism (Table 5; Fig. 2).

\subsection{Gut microbes in Altica species}

\subsubsection{Taxonomic classification and abundance composition of microbes in Altica}

On average, we predicted $117,464\left(65,305^{\sim} 155,268\right)$ genes per sample in the Altica gut microbiota (Table 6). Among the 312,458 non-redundant genes that were identified, $30.5 \%(95,369)$ matched the $\mathrm{Nr}$ database. Sequences from Archaea accounted for $1.91 \%, 59.26 \%$ were bacteria, $34.82 \%$ were fungi, and $4.01 \%$ of the sequences were viruses (Table 6).

The meta-transcriptome results indicated that there is a rich diversity of microbes in Altica digestive tracts. In total, we identified 6,435 species representing 1,950 genera, 687 families, 298 orders, 137 Classes and 127 phyla in at least one sample (Table 7). Among the groups identified, bacteria dominanted the samples. We found 106 phyla in the guts of Altica species (excluding unclassified or unidentified taxa), with the most abundant phyla being Proteobacteria (54.0\%), Actinobacteria (21.12\%), Firmicutes (9.69\%), Bacteroidetes (5.79\%), Chloroflexi $(2.47 \%)$ and Acidobacteria (1.25\%). The next most diverse group was fungi where we identified eight phyla, including Ascomycota (35.48\%), Mucoromycota (26.14\%), Chytridiomycota (12.63\%) and Zoopagomycota (11.36\%). There were 12 phyla of Archaea, with the most abundant ones being Euryarchaeota $(65.22 \%)$ and Thaumarchaeota $(15.03 \%)$, and there was a single phylum of viruses, Negarnaviricota (Table S3).

Generic diversity was similarly high. In total, there were 1,400 genera of bacteria that were identified among the 12 pooled samples, with the majority of sequences ( $>2 \%$ of all bacterial sequences) being from a few genera:Bdellovibrio(11.91\%),Enterococcus(6.78\%), Acinetobacter (6.57\%), Macrococcus (6.17\%), Microbacterium (6.16\%), Streptomyces (5.98\%), Escherichia (5.46\%), Enterobacter (3.86\%), Thalassobius (2.97\%), Kangiella $(2.48 \%)$ and Pseudomonas (2.33\%). We found a total of 408 genera of fungi, consisting primarily of ( $>2 \%$ of all fungal sequences)Basidiobolus (6.84\%), Aspergillus (6.29\%), Allomyces (5.63\%), Spizellomyces (4.70\%),Syn- 
cephalastrum (3.61\%), Geotrichum (2.94\%),Mortierella (2.91\%), Lobosporangium (2.85\%),Batrachochytrium (2.61\%), Sphaerulina (2.46\%), Rhizopus (2.38\%), Rozella (2.25\%) and Puccinia(2.14\%). The Archae sequences were from 48 genera, mainly including ( $>2 \%$ of all Archaea sequences) Halosimplex (69.62\%), Candidatus Methanodesulfokores (9.35\%),Nitrosopumilus (7.31\%), Methanosarcina (2.92\%) and Candidatus Nitrosocosmicus (2.50\%). The viruses belonged to 91 genera, including ( $>2 \%$ of all the Virus sequences)Ascovirus (24.11\%), Bracovirus (17.74\%), Alphabaculovirus (8.73\%), Alpharetrovirus (6.65\%), Invictavirus (5.91\%), Chlorovirus (5.11\%), Errantivirus (4.25\%), Gammaretrovirus (3.82\%), Alphaentomopoxvirus (2.82\%), Avipoxvirus (2.39\%), and Hepacivirus (2.38\%) (Table S4).

We were able to assign species identities to many of the sequences. For example, the bacterial sequences were assigned to 5,078 species, including Gammaproteobacteria_bacterium_2W06 (29.41\%), Enterococcus faecium (4.39\%), Macrococcus caseolyticus(4.06\%), Microbacterium arborescens (4.01\%), Acinetobacter _sp._WCHAc060041 (3.78\%), Escherichia coli(3.55\%), and Streptomyces tsukubensis (3.25\%). There were 812 species of Fungi identified, with the most abundant species beingBasidiobolus meristosporus (6.94\%), Allomyces macrogynus(5.71\%), Spizellomyces punctatus (4.77\%), Syncephalastrum racemosum (3.66\%), Geotrichum candidum (2.98\%), Lobosporangium transversale (2.89\%), Sphaerulina musiva (2.51\%), Rozella allomycis (2.29\%), and Aspergillus saccharolyticus (2.16\%). The Archaea sequences were assigned to 165 species, the most abundant species being Halosimplex carlsbadense (30.22\%), Euryarchaeota archaeon (18.07\%), Thaumarchaeota archaeon_13_1_40CM_2_39_4 (6.99\%), Methanophagales archaeon (6.44\%), and Candidatus_Verstraetearchaeota archaeon $(6.43 \%)$. Herein 380 virus species were identified, the most abundant ones are Cotesia_sesamiae_bracovirus (12.90\%), Solenopsis_invicta_virus_3 (5.63\%) and Hubei_tombus-like_virus_$16(5.99 \%)$ (Table S5).

\subsubsection{Alpha-diversity}

There were significant differences in alpha -diversity in comparisons of the pooled data (i.e., Archaea, bacteria, fungi and viruses) among the four feeding treatmeats (AFDi, AVGn, $\mathrm{F}_{1} \mathrm{Di}, \mathrm{F}_{1} \mathrm{Gn}$ ) (Table 8). We also found significant differences in the ACE and Chaol indicators when we compared all beetles that were fed the two host plants (i.e., DiT vs. GnT). Similar patterns were detected when we only considered the bacterial data (Table 8). For the fungal dataset, there were significant differences in alpha -diversity among the four treatmeats for three of the indicators (ACE, Chao1 and Simpson) whereas there were no differences among groups when we pooled the beetles fed the two host plants (Table 8).

\subsubsection{Beta-diversity}

The PCoA on the pooled data (Archaea, bacteria, fungi and viruses) showed that the four treatments were distributed in four quadrants (Fig 3). The first principal coordinate explained $57.5 \%$ (Bray-Curtis) and 49.9\% (Jaccard) of the total variance and separated samples by host plant (Fig. 3). The NMDS analysis showed similar patterns as the PCoA (results not shown). Both ANOSIM and PerMANOVA identified significant differences among the four treatments (ANOSIM: Jaccard $\mathrm{p}<0.002$, Bray-Curtis $\mathrm{p}<0.001$; PerMANOVA: Jaccard $\mathrm{p}<0.001$, Bray-Curtis, $\mathrm{p}<0.001)$. Furthermore, significant diffenrences were always detected when we considered different datasets (pooled, bacterial or fungal data), distance methods (Bray-Curtis or Jaccard index) or statistical methods (PerMANOVA or ANOSIM) (Table 9). In addition, the results of two-way PerMANOVA using beetle species and plant diet as main effects showed that both of these factors played notable roles in structuring gut microbial communities withinAltica species (Table 10).

\subsubsection{Taxonomic composition comparisons}

There were significant differences in taxonomic composition associated with both beetle group and plant diet (Table 11). For example, in the comparison of the two parental beetles species, AFDi vs. AVGn, there were significant differences in relative abundance in the pooled (546 genera and 1489 species), bacterial (423 genera and 1335 species), and fungal (112 genera and 213 species) datasets. Similarly, in the comparisons of the F1 hybrids fed different host plant species and the pooled comparison of beetles fed the different plant species, there were also significant differences in relative abundance across the different datasets (Table 11; Table S6). 


\subsubsection{Functional diversity of gut microbes}

Gene functional annotation

After assembly, there was a mean of 117,646 $( \pm 2,992)$ genes predicted per sample (Table 12). In total, we identified 312,458 non-redundant genes, and 95,369 of these genes were annotated to the Nr database. On average, we annotated 3,558 $( \pm 389)$ orthologs and $152( \pm 5)$ pathways to the KEGG database, and 10,343 $( \pm 1,355)$ genes to the eggNOG database (Table 13).

\section{Beta-diversity of community function}

Based on the KEGG Orthology (KO) dataset, a PCA revealed clear separation of the different beetle and plant diet groups. The first principal component explained $82.8 \%$ of the total variance and separated the samples by plant diet, and the second principal component explained $12.0 \%$ of the variance (Fig 4). The PCA based on eggNOG Orthology showed highly similar groupings, as did the PCoA and NMDS analyses (results not shown). Both ANOSIM and PerMANOVA indicated that there are significant differences among the four treatment groups (AFDi, AVGn, $\mathrm{F}_{1} \mathrm{Di}, \mathrm{F}_{1} \mathrm{Gn}$ ) and also between the two pooled groups of beetles fed different plant diets (Table 14).

Differentially expressed genes and KEGG functional enrichment

Using edgeR analysis, we found a number of genes that exhibited significant differential expression (FDR p $<$ 0.05) in our comparisons of beetles groups and plant diet (Table 15, Table S7). In total, 17,390 non-redundant genes were annotated to the KEGG database, and 3,473, 1,204 and 436 genes showed differential expression in the comparisons of AFDi vs. AVGn, DiT vs. GnT and $F_{1}$ Di vs. $F_{1}$ Gn, respectively (Table 15). Among these, we identified 1895 differentially expressed genes in KEGG pathways in the comparison of AFDi vs. AVGn, 764 in the comparison of DiT vs. GnT, and 298 differentially expressed genes in the comparison of $\mathrm{F}_{1}$ Di vs. $\mathrm{F}_{1}$ Gn (Table S7). Likewise, the KEGG functional enrichment analysis revealed several pathways that were significantly enriched in the comparisons (Table 16).

\section{DISCUSSION}

A number of studies have suggested that host plant species is one of the major drivers shaping gut microbiota in herbivorous insects (e.g., Gayatri Priya et al., 2012; Mason et al., 2015; Berasategui et al., 2016; Chung et al., 2017; Strano et al., 2018; Jones et al., 2019). Here we set out to examine (1) differences in community diversity and composition of gut microbes and (2) predict the role of insect-derived versus gut microbiotaderived detoxification functions in two specialist Altica flea beetles and their $\mathrm{F}_{1}$ hybrids. Previous work in this system that compared field-collected adult beetles indicated that there was little species-specific patterns of gut bacterial communities between Altica species that were feeding on different host plant species (Wei et al., 2020). Instead, we observed significant differentiation of microbial communities caused by geographic location, suggesting that these beetles are obtaining their gut communities locally. Any host-specific patterns may have been masked by relatively high variability in bacterial composition among geographically separated populations (Wei et al., 2020).

In contrast with these previous findings, we used a detailed metatranscriptomic analysis to show that both beetle species identity and diet impact diversity and composition of the gut microbial communities of Altica - Beta-diversity analyses consistently showed significant differences beteween treatments no matter how the data were parsed (i.e., pooled, bacterial versus fungal data), no matter the metric used (i.e., Bray-Curtis or Jaccard index) and irrespective of the statistical approach (i.e., PerMANOVA or ANOSIM) (Table 9). In addition, a two-way PerMANOVA also indicated that both host species and host diet had a significant effect on the gut microbial community (Table 10). Perhaps the most convincing evidence of diet impacting gut microbial communities in Altica are the results for the $\mathrm{F}_{1}$ hybrids. In this comparision, the only difference between the beetle groups was diet, thus providing direct support for the idea that beetle diet dictates gut microbial community composition.

These results were somewhat surprising because in the present study, there were only two main sources from 
which the lab-reared beetles could obtain their gut microbes: the host plant materials fed to the beetles and the laboratory environment. Both of these microbial sources should have favored similar gut microbial communities. For example, the host plant tissues were collected from the same sympatric site, albeit they were collected from different host plant species; thus, the beetles were acquiring their gut microbiota from a common environmental source. Furthermore, the beetles were maintained in the same insectary, so the small proportion of gut microorganisms that came from the laboratory environment should have also been shared. Thus, the question remains as to why we observed differences in gut microbial community diversity and composition.

One possible explanation for the differences in gut microbial communities is that there are differences in the beetle host species that determine community composition. For instance, there could be selective factors that differ between Altica species such as gut $\mathrm{pH}$, digestive enzymes, ionic strength and redox potential of gut compartments (Tang et al., 2012; Engel \& Moran, 2013; Martinson et al., 2017; Ravenscraft et al., 2019) that alter gut microbial communities. Alternatively, since we did not sequence the microorganisms present on the plant materials, it is unknown whether differences in the chemistry of the plant species filtered the environmental microbiota encountered by the beetles. Although this is possible, past research has shown that changes in the concentrations of defense chemicals, such as phenolic glycosides and condensed tannins do not influence the composition of foliar microbial communities (Mason et al., 2015). A key next step in resolving this question will be to examine the foliar microbial communities of Altica host plants.

In addition to observing differences in microbial diversity and composition, we also identified a number of differentially expressed genes in comparisons of the beetle species and feeding treatments. We found 1613 DEGs in comparisons of the two Altica species, and when we compared the two diet treatment groups for the $\mathrm{F}_{1}$ hybrids, there were 164 DEGs detected. Among these DEGs, a noteworthy proportion were detoxificationrelated genes that included P450s, CCEs, GST, UGTs, and ABCs (Table S2). Many of these differentially expressed genes have been shown to be related to host plant species use and/or chemistry (e.g., Wybouw et al., 2015; Zhu et al., 2016; Zhong et al., 2017; Scully et al., 2018; Huang et al., 2020), suggesting that differential expression of these genes may be associated with the host plant species on which the beetles are feeding. Particularly in the comparison of the $F_{1}$ hybrids, this suggests a response to the diet treatment that is either mediated by plasticity or by the host plant materials filtering microbial species composition.

Similar to other insect species (e.g., Celorio-Mancera et al., 2012), the Altica gut DEGs between different plant-feeding treatments were enriched in lysosome, glutathione metabolism and several kinds of amino acid metabolism pathways based on the KEGG database (Table 5). Pathways for lysosome enzymes, tryptophan metabolism (Terra et al., 2018), drug metabolism-cytochrome P450 and ABC transporters have been suggested to be related to secondary compound detoxification (Koenig et al., 2015; Després et al., 2007; Ahn et al., 2012; Dermauw \& Van Leeuwen, 2014; Merzendorfer, 2014; Rane et al., 2016). These DEGs were mainly enriched in amino acid metabolism, lysosome enzymes, glutathione metabolism and tryptophan metabolism pathways (Table 5). Among these DEGs, glutathione metabolism has been suggested to be involved in detoxification and oxidation resistance (Yang et al., 2018), whereas lysosome pathways, tryptophan metabolism (Terra et al., 2018), drug metabolism and ABC transporters have also been suggested to be related to digestion and detoxification. When we focused on the subset of the data associated with gut microbes, we observed that microbial DEGs were primarily enriched in glutathione metabolism; however, there were a number of other pathways that were also enriched. Notably, we saw enrichment of cyanoamino acid metabolism, geraniol metabolism, arachidonic acid metabolism, benzoate metabolism, carbon metabolism, aromatic compound metabolism, tryptophan metabolism and fatty acid metabolism pathways (Table 16). Among this set of pathways, a number have been related to plant secondary compound metabolism, such as glutathione metabolism, geraniol metabolism, aromatic compound metabolism and tryptophan metabolism, suggesting that both the beetles and gut microbes are enriched in detoxification pathways that could help the beetles contend with plant secondary chemistry.

The idea that microbes are contributing to digestion and detoxification of Altica host plant material is further supported by the species composition of the microbial communities. For example, we know that 
there is a complex array of plant secondary metabolites present in both of the host plants used in this study, including more than 110 compounds identified in Duchesnea indica (Li et al., 2017) and over 180 compounds in Geranium species (Yang et al., 2015). The major secondary metabolites include triterpenoids, flavonoids, phenolic acids, ellagic acids, glycosides, essential oils, lignins, and sterols (Ye \& Yang, 1996; Lei et al., 2002; Xu et al., 2007; He et al., 2011; Yang et al., 2015; Li et al., 2017), and many, if not all, of these can be degraded by microorganisms. For instance, flavonoids can be degraded by fungi (Aspergillus niger, Acremonium ) and bacteria (Eubacterium , Clostridium orbiscindens ) (Sakai, 1977; Schneider \& Blaut, 2000; Blaut et al., 2003; Schoefer et al., 2003; Weiz et al., 2019); tannins can be degraded by a number of bacteria (Enterobacter, Cellulomonas, Staphylococcus ,Arthrobacter, Bacillus, Micrococcus ,Corynebacterium, Pseudomonas, Klebsiella) and fungi (Aspergillus, Penicillium, Chaetomium, Fusarium, Rhizoctonia, Trichoderma) (Bhat et al., 1998). We also know that ursolic acid can be degraded by the fungus Syncephalastrum racemosum (Huang et al., 2012), terpenes can be degraded by fungi (Grosmannia) and many bacteria (Brevundimonas, Caulobacter, Pseudomonas, Rahnella, Serratia, Burkholderia, Rhodococcus , Nocardia ,Geobacillus, Mycobacterium, Novosphingobium, Corynebacterium, Sphingomonas, Lactobacillus ,Castellaniella ) (Parra et al., 2009; Adams et al., 2013; Marmulla \& Harder 2014). In addition, phenolic acid is degraded byBifidobacterium (Fritsch et al., 2016) and glycosides by Acinetobacter (Mason et al., 2014).

Interestingly, this broad diversity of microbes that have been shown to degrade plant secondary chemistry were all identified in the guts of Altica species in the present study, suggesting a functional role for their speciose gut microbiota that may provide the material basis for helping these beetles to use their host plants. Indeed, some of these microbes accounted for a high proportion of the microbial fauna. For example, the bacterium Acinetobacter and fungusAspergillus each accounted for about $6 \%$ of the microbes present in the samples. Furthermore, some of these microbes associated with plant secondary degradation also showed significant diferences in relative abundance between the feeding treatments, such asAcinetobacter, Acremonium, Eubacterium, Staphylococcus, Arthrobacter, Bacillus, Corynebacterium, Sphingomonas and Lactobacillus (Table S6). Although it is likely that many of the species are contributing to detoxification of host plant secondary chemistry, the evidence presented in this study is not definitive. Functional studies on candidate microbes are still needed to provide direct support for the role of these microbes in Altica digestion. Thus far, the results are consistent with the view that Altica may be benefiting from the degradation and detoxification actions of their gut microbes.

The results also underscore the power of using meta-transcriptomic approaches to examine the role and function of gut microbes in insect digestion. This deep sequencing method revealed a striking diversity of gut symbionts in Altica beetles that identified an average of 2,855 bacterial and 752 fungal species per sample (Table 7). Recent advances in "-omics" analyses have shown that the diversity in microbial populations is significantly higher than previously estimated by traditional culturing and molecular methods, suggesting that dominant microorganisms identified with these approaches may mask the rare species (Yun et al., 2014). Consistent with this suggestion, many of the microbes within our samples were rare, and only 987 species were present at greater than $0.01 \%$ abundance. Our previous work using $16 \mathrm{~S}$ rRNA sequencing only identified 343 bacterial OTUs from the guts of threeAltica species (Wei et al., 2020), indicating that deep sequencing approaches facilitated identification of the rare microbial species present at low abundance in the samples.

The differences in the number of microbial taxa identified between our two studies of Altica gut microbiomes suggests that we may be underestimating the number of species in insect guts. Most studies of insect gut microbiomes have been carried out using $16 \mathrm{~S}$ rRNA gene amplicon sequence data, and these studies have shown considerable variation among insect species. For example, more than 1,000 bacterial operational taxonomic units (OTUs) were identified from the wood-feeding beetle Prionoplus reticularis (Reid et al., 2011), twoPachysoma dung beetle species (Franzini et al., 2016), and the pine processionary moth Thaumetopoea pytiocampa (Strano et al., 2018). In comparison, about 250 bacterial OTUs were detected from the pine weevil Hylobius abietis (Berasategui et al., 2016) and the Asian longhorned beetle Anoplophora glabripennis (Scully et al., 2018), whereas less than 200 bacterial species were identified inHyles euphorbiae , Brithys crini (Vilanova et al., 2016), Plutella xylostella (Xia et al., 2017) and the ladybird beetleHarmonia axyridis (Dudek et al., 2017). It would be interesting to determine whether this variability among species represents 
functional shifts in microbial diversity and if microbial diversity estimates generally change drastically when using deep sequencing approaches.

It was suggested that foliar-feeding insects acquire microbiomes from the plant materials and also from soil via host plants (Hannula et al., 2019). Both Duchesnea indica and Geranium nepalense are short herbaceous plants, the soil microbiomes are easy to transfer to adjacent leaf tissue, so it is possible for these two Alticaspecies to acquire microbiomes from soil. Therefore the huge biodiversity of soil microbes could partly explain the high biodiversity of beetle gut microbiomes in the present study.

Together, the results presented here suggest that host plant species use can influence insect gut gene expression, gut microbial community structure, and gut microbial gene expression in Altica species. The results support the idea that beetles deal with plant secondary compounds by using plastic transcriptional responses and by recruiting microorganisms that can assist them with the digestion of host plant tissues. Consequently, Altica beetles may be cooperating with their gut microbiota, and in doing so, this may allow them to switch to new host plants and contribute to the process of host associated diversification.

\section{ACKNOWLEDGEMENTS}

This study was supported by National Natural Science Foundation of China (Grant No. 31672334, 31472030). We thank the Biomarker Technologies Corporation (Beijing, China) for conducting the meta-sequencing.

\section{REFERENCES}

[dataset] Wei, J,; Yang, X. K.; Segraves, K. A.; Xue, H. J.; 2021; meta-transcriptome seqences of Altica guts; National Genomics Data Center (https://bigd.big.ac.cn/gsa).; Persistent identifier XXXXXX.

Adams, A. S., Aylward, F. O., Adams, S. M., Erbilgin, N., Aukem, B. H., Currie, C. R., Suen, G., \& Raffaa, K. F. (2013). Mountain pine beetles colonizing historical and naïve host trees are associated with a bacterial community highly enriched in genes contributing to terpene metabolism. Applied and Environmental Microbiology , 61, 759-768. https://doi.org/10.1128/AEM.00068-13

Altschul, S. F., Gish, W., Miller, W., Myers, E. W., \& Lipman, D. J. (1990). Basic local alignment search tool. Journal of Molecular Biology , 215, 403-410. https://doi.org/10.1016/S0022-2836(05)80360-2

Anders, S., \& Huber, W. (2010). Differential expression analysis for sequence count data. Genome Biology , 11, R106. https://doi.org/10.1186/gb-2010-11-10-r106

Ashburner, M., Ball, C. A., Blake, J. A., Botstein, D., Butler, H., Cherry, J. M., Davis, A. P., Dolinski, K., Dwight, S. S., Eppig, J. T., Harris, M. A., Hill, D. P., Issel-Tarver, L., Kasarskis, A., Lewis, S., Matese, J. C., Richardson, J. E., Ringwald, M., Rubin G. M. \& Sherlock, G. (2000). Gene Ontology: tool for the unification of biology. Nature genetics , 25, 25-29. https://doi.org/10.1038/75556

Bairoch, A., \& Apweiler, R. (1996). The SWISS-PROT protein sequence data bank and its new supplement TREMBL. Nucleic Acids Research , 24, 21-25. https://doi.org/10.1093/nar/21.13.3093

Bashiardes, S., Zilberman-Schapira, G., \& Elinav, E. (2016). Use of metatranscriptomics in microbiome research. Bioinformatics and Biology Insights , 10, 19-25. https://doi.org/10.4137/BBI.S34610

Benjamini, Y., \& Hochberg, Y. (1995). Controlling the false discovery rate: a practical and powerful approach to multiple testing. Journal of the Royal Statistical Society: Series B (Methodological), 57, 289-300. https://doi.org/10.1111/j.2517-6161.1995.tb02031.x

Berasategui, A., Axelsson, K., Nordlander, G., Schmidt, A., Borg-Karlson, A., Gershenzon, J., Terenius, O., \& Kaltenpoth, M. (2016). The gut microbiota of the pine weevil is similar across Europe and resembles that of other conifer-feeding beetles. Molecular Ecology , 25, 4014-4031. https://doi.org/10.1111/mec.13702

Berasategui, A., Salem, H., Paetz, C., Santoro, M., Gershenzon, J., Kaltenpoth, M., \& Schmidt, A. (2017). Gut microbiota of the pine weevil degrades conifer diterpenes and increases insect fitness. Molecular Ecology , 26, 4099-4110. https://doi.org/10.1111/mec.14186 
Berenbaum, M. R., \& Zangerl, A. R. (1998). Chemical phenotype matching between a plant and its insect herbivore. Proceedings of the National Academy of Sciences of the United States of America, 95, 1374313748. https://doi.org/10.1073/pnas.95.23.13743

Bhat, T. K., Singh, B., \& Sharma, O. P. (1998). Microbial degradation of tannins - A current perspective. Biodegradation , 9, 343-357. https://doi.org/10.1023/A:1008397506963

Blaut, M., Schoefer, L., \& Braune, A. (2003). Transformation of flavonoids by intestinal microorganisms. International Journal for Vitamin and Nutrition Research , 73 (2), 79-87. https://doi.org/10.1024/03009831.73.2.79

Boone, C. K., Keefover-Ring, K., Mapes, A. C., Adams, A. S., Bohlmann, J., \& Raffa, K. F. (2013). Bacteria associated with a tree-killing insect reduce concentrations of plant defense compounds. Journal of Chemical Ecology , 39, 1003-1006. https://doi.org/10.1007/s10886-013-0313-0

Ceja-Navarro, J. A., Vega, F. E., Karaoz, U., Hao, Z., Jenkins, S., Lim, H. C., Kosina, P., Infante, F., Northen, T. R., \& Brodie, E. L. (2015). Gut microbiota mediate caffeine detoxification in the primary insect pest of coffee. Nature Communications , 6, 7618. https://doi.org/10.1038/ncomms8618

Celorio-Mancera, M. d. l. P., Heckel, D. G., \& Vogel, H. (2012).Transcriptional analysis of physiological pathways in a generalist herbivore: responses to different host plants and plant structures by the cotton bollworm, Helicoverpa armigera .Entomologia Experimentalis et Applicata , 144, 123-133. https://doi.org/10.1111/j.1570-7458.2012.01249.x

Chong, J., Liu, P., Zhou, G., \& Xia, J. (2020). Using MicrobiomeAnalyst for comprehensive statistical, functional, and meta-analysis of microbiome data. Nature Protocols , 15, 799-821. https://doi.org/10.1038/s41596-019-0264-1

Chu, C. C., Spencer, J. L., Curzi, M. J., Zavala, J. A., \& Seufferheld, M. J. (2013). Gut bacteria facilitate adaptation to crop rotation in the western corn rootworm. Proceedings of the National Academy of Sciences of the United States of America , 110, 11917-11922. https://doi.org/10.1073/pnas.1301886110

Chung, S. H., Scully, E. D., Peiffer, M., Geib, S. M., Rosa, C., Hoover, K., \& Felton, G. W. (2017). Host plant species determines symbiotic bacterial community mediating suppression of plant defenses.Scientific Reports , 7, 39690. https://doi.org/10.1038/srep39690

Colman, D. R., Toolson, E. C., \& Takacs-Vesbach, C. D. (2012). Do diet and taxonomy influence insect gut bacterial communities? Molecular Ecology , 21, 5124-5137. https://doi.org/10.1111/j.1365-294X.2012.05752.x

De Fine Licht, H. H., Schiott, M., Rogowska-Wrzesinska, A., Nygaard, S., Roepstorff, P., \& Boomsma, J. J. (2012). Laccase detoxification mediates the nutritional alliance between leaf-cutting ants and fungusgarden symbionts. Proceedings of the National Academy of Sciences of the United States of America, 110, 583-587. https://doi.org/10.1073/pnas.1212709110

Dermauw, W. \& Van Leeuwen, T. (2014). The ABC gene family in arthropods: Comparative genomics and role in insecticide transport and resistance. Insect Biochemistry and Molecular Biology , 45, 89-110. https://doi.org/10.1016/j.ibmb.2013.11.001

Després, L., David, J. P., \& Gallet, C. (2007). The evolutionary ecology of insect resistance to plant chemicals. Trends in Ecology \&3 Evolution , 22, 298-307. https://doi.org/10.1016/j.tree.2007.02.010

Dhariwal, A., Chong, J., Habib, S., King, I., Agellon, L. B., \& Xia, J. (2017). MicrobiomeAnalyst - a web-based tool for comprehensive statistical, visual and meta-analysis of microbiome data. Nucleic Acids Research, 45, W180-188. https://doi.org/10.1093/nar/gkx295

Douglas, A. E. (2015). Multiorganismal insects: diversity and function of resident microorganisms. Annual Review of Entomology , 60, 17-34. https://doi.org/10.1146/annurev-ento-010814-020822 
Dudek, K., Humińska, K., Wojciechowicz, J., \& Tryjanowski, P. (2017). Metagenomic survey of bacteria associated with the invasive ladybird Harmonia axyridis (Coleoptera: Coccinellidae). European Journal of Entomology , 114, 312-316. https://doi.org/10.14411/eje.2017.038

Engel, P., \& Moran, N. A. (2013). The gut microbiota of insects diversity - in structure and function.FEMS Microbiology Reviews , 37, 699-735. https://doi.org/10.1111/1574-6976.12025

Finn, R. D., Bateman, A., Clements, J., Coggill, P., Eberhardt, R. Y., Eddy, S. R., Heger, A., Hetherington, K., Holm, L., Mistry, J., Sonnhammer, E. L. L., Tate, J. \& Punta, M. (2014). Pfam: the protein families database. Nucleic Acids Research , 42, D222-D230. https://doi.org/10.1093/nar/gkt1223

Forbes, A.A., Devine, S. N., Hippee, A. C., Tvedte, E. S., Ward, A. K. G., Widmayer, H. A., \& Wilson, C. J. (2017). Revisiting the particular role of host shifts in initiating insect speciation. Evolution , 71, 1126-1137. https://doi.org/10.1111/evo.13164

Franzini, P. Z. N., Ramond, J. B., Scholtz, C. H., Sole, C. L., Ronca, S., \& Cowan, D. A. (2016). The gut microbiomes of two pachysoma Macleay desert dung beetle species (Coleoptera: Scarabaeidae: Scarabaeinae) feeding on different diets. PLoS One, 11, e0161118. https://doi.org/10.1371/journal.pone.0161118

Fritsch, C., Heinrich, V., Vogel, R. F., \& Toelstede, S. (2016). Phenolic acid degradation potential and growth behavior of lactic acid bacteria in sunflower substrates.Food Microbiology , 57, 178-186. https://doi.org/10.1016/j.fm.2016.03.003

Fu, L. M., Niu, B. F., Zhu, Z. W., Wu, S. T., \& Li, W. Z. (2012). CD-HIT: accelerated for clustering the nextgeneration sequencing data.Bioinformatics , 28, 3150-3152. https://doi.org/10.1093/bioinformatics/bts565

Gayatri Priya, N., Ojha, A., Kajla, M. K., Raj, A., \& Rajagopal, R. (2012). Host plant induced variation in gut bacteria of Helicoverpa armigera . PLoS One, 7, e30768. https://doi.org/10.1371/journal.pone.0030768

Gifford, S. M., Sharma, S., Rinta-Kanto, J. M., \& Moran, M. A. (2011). Quantitative analysis of a deeply sequenced marine microbial metatranscriptome. The ISME Journal , 5, 461-472. https://doi.org/10.1038/ismej.2010.141

Grabherr, M. G., Haas, B. J., Yassour, M., Levin, J. Z., Thompson, D. A., Amit, I., Adiconis, X., Fan, L., Raychowdhury, R., Zeng, Q. D., Chen, Z. H., Mauceli, E., Hacohen, N., Gnirke, A., Rhind, N., di Palma, F., Birren, B. W., Nusbaum, C., Lindblad-Toh, K., Friedman, N., \& Regev, A. (2011). Full-length transcriptome assembly from RNA-Seq data without a reference genome. Nature Biotechnology , 29, 644-652. https://doi.org/10.1038/nbt.1883

Hammer, Ø., Harper, D., \& Ryan, P. (2001). PAST: Paleontological statistics software package for education and data analysis. Palaeontol Electron , 4, 1-9.

Hammer, T. J., \& Bowers, M. D. (2015). Gut microbes may facilitate insect herbivory of chemically defended plants. Oecologia , 179, 1-14. https://doi.org/10.1007/s00442-015-3327-1

Hammer, T. J., Janzen, D. H., Hallwachs, W., Jaffe, S. P., \& Fierer, N. (2017). Caterpillars lack a resident gut microbiome. Proceedings of the National Academy of Sciences of the United States of America, 114, 9641-9646. https://doi.org/10.1073/pnas.1707186114

Hannula, S. E., Zhu, F., Heinen, R., \& Bezemer, T. M. (2019). Foliar-feeding insects acquire microbiomes from the soil rather than the host plant.Nature Communications , 10, 1254. https://doi.org/10.1038/s41467019-09284-w

Hansen, A. K., \& Moran, N. A. (2014). The impact of microbial symbionts on host plant utilization by herbivorous insects. Molecular Ecology , 23, 1473-1496. https://doi.org/10.1111/mec.12421

He, W. T., Jin, Z. X., \& Wang, B. Q. (2011). Research progress of Geranium nepalense Sweet. Journal of Aerospace Medicine, 21, 1200-1202. 
Huang, F. X., Yang, W. Z., Ye, F., Tian, J. Y., Hu, H. B., Feng, L. M., Guo, D. A., \& Ye, M. (2012). Microbial transformation of ursolic acid by Syncephalastrum racemosum (Cohn) Schroter AS 3.264. Phytochemistry , 82, 56-60. https://doi.org/10.1016/j.phytochem.2012.06.020

Huang, H. J., Cui, J. R., \& Hong, X. Y. (2020). Comparative analysis of diet-associated responses in two rice planthopper species. BMC Genomics , 21, 565. https://doi.org/10.1186/s12864-020-06976-2

Itoh, H., Tago, K., Hayatsu, M., \& Kikuchi, Y. (2018). Detoxifying symbiosis: microbe-mediated detoxification of phytotoxins and pesticides in insects. Natural Product Reports , 35, 434-454. https://doi.org/10.1039/c7np00051k

Jones, A. G., Mason, C. J., Felton, G. W., \& Hoover, K. (2019). Host plant and population source drive diversity of microbial gut communities in two polyphagous insects. Scientific Reports , 9, 2792. https://doi.org/10.1038/s41598-019-39163-9

Kanehisa, M., Goto, S., Kawashima, S., Okuno, Y., \& Hattori, M. (2004). The kegg resource for deciphering the genome. Nucleic Acids Research , 32, D277-D280. https://doi.org/10.1093/nar/gkh063

Kim, D., Pertea, G., Trapnell, C., Pimentel, H., Kelley, R., \& Salzberg, S. L. (2013). TopHat2: accurate alignment of transcriptomes in the presence of insertions, deletions and gene fusions. Genome Biology , 14, R36. https://doi.org/10.1186/gb-2013-14-4-r36

Koenig, C., Bretschneider, A., Heckel, D. G., Grosse-Wilde, E., Hansson, B. S., \& Vogel, H. (2015). The plastic response of Manduca sexta to host and non-host plants.Insect Biochemistry and Molecular Biology, 63, 72-85. https://doi.org/10.1016/j.ibmb.2015.06.001

Langmead, B., \& Salzberg, S. L. (2012). Fast gapped-read alignment with Bowtie 2. Nature Methods , 9, 357359. https://doi.org/10.1038/nmeth.1923

Lei, Z. Y., Liu, D. L., Hu, Y. Q., \& Cui, L. G. (2002). Research progress on chemical compositions and pharmacology of Geranium species. Journal of Chinese Medicinal Materials , 25, 759-761.

Li, M., He, J., Ma, B. Z., Liang, Y. Y., Zhang, S. J., Zhang, X. L., \& Cui, G. (2017). Research progress on chemical compositions and anticancer pharmacology of the traditional Chinese herb Duchesnea indica . Evaluation and Analysis of Drug-use in Hospitals of China , 17, 595-600.

Love, M. I., Huber, W., \& Anders, S. (2014). Moderated estimation of fold change and dispersion for RNA-Seq data with DESeq2. Genome Biology , 15, 550. https://doi.org/10.1186/s13059-014-0550-8

Mao, X. Z., Cai, T., Olyarchuk, J. G., \& Wei, L. P. (2005) Automated genome annotation and pathway identification using the KEGG Orthology (KO) as a controlled vocabulary. Bioinformatics , 21, 3787-3793. https://doi.org/10.2307/1592215

Marmulla, R., \& Harder, J. (2014). Microbial monoterpene transformations-a review. Frontiers in Microbiology , 5, 346. https://doi.org/10.3389/fmicb.2014.00346

Martinson, V. G., Douglas, A. E., \& Jaenike, J. (2017). Community structure of the gut microbiota in sympatric species of wild Drosophila . Ecology Letters , 20, 629-639. https://doi.org/10.1111/ele.12761

Mason, C. J., Couture, J. J., \& Raffa, K. F. (2014). Plant-associated bacteria degrade defense chemicals and reduce their adverse effects on an insect defoliator. Oecologia , 175, 901-910. https://doi.org/10.1007/s00442014-2950-6

Mason, C. J., Rubert-Nason, K. F., Lindroth, R. L., \& Raffa, K. F. (2015). Aspen defense chemicals influence midgut bacterial community composition of gypsy moth. Journal of Chemical Ecology , 41, 75-84. https://doi.org/10.1007/s10886-014-0530-1

Merzendorfer, H. (2014). ABC Transporters and their role in protecting insects from pesticides and their metabolites. Advances in Insect Physiology , 46, 1-72. https://doi.org/10.1016/B978-0-12-417010-0.00001-X 
Näsvall, K., Wiklund, C., Künstner, A., Talla, V., Busch, H., Vila, R., \& Backström, N. (2021). Host plant diet affects growth and induces altered gene expression and microbiome composition in the wood white (Leptidea sinapis ) butterfly. Molecular Ecology , 30, 499-516. https://doi.org/10.1111/mec.15745

Orsucci, M., Audiot, P., Dorkeld, F., Pommier, A., Vabre, M., Gschloessl, B., Rialle, S., Severac, D., Bourguet, D., \& Streiff, R. (2018). Larval transcriptomic response to host plants in two related phytophagous lepidopteran species: implications for host specialization and species divergence. BMC Genomics , 19, 265. https://doi.org/10.1186/s12864-018-4589-x

Parra, A., Rivas, F., Garcia-Granados, A., \& Martinez, A. (2009). Microbial transformation of triterpenoids. Mini-reviews in Organic Chemistry , 6, 307-320.

Powell, S., Forslund, K., Szklarczyk, D., Trachana, K., Roth, A., Huerta-Cepas, J., Gabaldón, T., Rattei, T., Creevey, C., Kuhn, M., Jensen, L. J., von Mering, C., \& Bork, P. (2014). eggNOG v4.0: nested orthology inference across 3686 organisms. Nucleic Acids Research , 42, D231-D239. https://doi.org/10.1093/nar/gkt1253

Pruitt, K. D., Tatusova, T., \& Maglott, D. R. (2007). NCBI reference sequences (RefSeq): a curated nonredundant sequence database of genomes, transcripts and proteins. Nucleic Acids Research, 35, D61-D65. https://doi.org/10.1093/nar/gki025

Rane, R. V., Walsh T. K., Pearce, S. L., Jermin, L. S., Goron, K. H. J., Richards, S., \& Oakeshott, J. G. (2016). Are feeding preferences and insecticide resistance associated with the size of detoxifying enzyme families in insect herbivores? Current Opinion in Insect Science , 13, 70-76. https://doi.org/10.1016/j.cois.2015.12.001

Ravenscraft, A., Berry, M, Hammer, T., Peay, K., \& Boggs, C. (2019). Structure and function of the bacterial and fungal gut microbiota of Neotropical butterflies. Ecological Monographs , 89, e01346. https://doi.org/10.1002/ecm.1346

Reid, N. M., Addison, S. L., Macdonald, L. J., \& Lloyd-Jones, G. (2011). Biodiversity of active and inactive bacteria in the gut flora of wood-feeding huhu beetle larvae (Prionoplus reticularis ). Applied Environmental Microbiology , 77, 7000-7006. https://doi.org/10.1128/AEM.05609-11

Rho, M., Tang, H. X., \& Ye, Y. Z. (2010). Fraggenescan: predicting genes in short and error-prone reads. Nucleic Acids Research, 38, e191. https://doi.org/10.1093/nar/gkq747

Sakai, S. (1977). Degradation of the plant flavonoid phellamurin by Aspergillus niger. Applied and Environmental Microbiology , 34, 500-505.

Scheffers, B. R., Joppa, L. N., Pimm, S. L., \& Laurance, W. F. (2012). What we know and don't know about Earth's missing biodiversity.Trends in Ecology Ef Evolution , 27, 501-510. https://doi.org/10.1016/j.tree.2012.05.008

Schneider, H., \& Blaut, M. (2000). Anaerobic degradation of flavonoids by Eubacterium ramulus . Archives of Microbiology , 173, 71-75. https://doi.org/10.1007/s002030050010

Schoefer, L., Mohan, R., Schwiertz, A., Braune, A., \& Blaut, M. (2003). Anaerobic degradation of flavonoids by Clostridium orbiscindens. Applied and Environmental Microbiology , 69, 5849-5854. https://doi.org/10.1128/AEM.69.10.5849-5854.2003

Scully, E. D., Geib, S. M., Mason, C. J., Carlson, J. E., Tien, M., Chen, H. Y., Harding, S., Tsai, C. J., \& Hoover, K. (2018). Host-plant induced changes in microbial community structure and midgut gene expression in an invasive polyphage (Anoplophora glabripennis ).Scientific Reports , 8, 9620. https://doi.org/10.1038/s41598-018-27476-0

Shukla, S. P., \& Beran, F. (2020). Gut microbiota degrades toxic isothiocyanates in a flea beetle pest. Molecular Ecology , 29, 4692-4705. https://doi.org/10.1111/mec.15657 
Simon, J., d'Alencon, E., Guy, E., Jacquin-Joly, E., Jaquiery, J., Nouhaud, P., Peccoud, .J, Sugio, A., \& Streiff, R. (2015). Genomics of adaptation to host-plants in herbivorous insects. Briefings in Functional Genomics , 14, 413-423. https://doi.org/10.1093/bfgp/elv015

Staudacher, H., Kaltenpoth, M., Breeuwer, J. A. J., Menken, S. B. J., Heckel, D. G., \& Groot, A. T. (2016). Variability of bacterial communities in the mothHeliothis virescens indicates transient association with the host. PLoS One, 11, e0154514. https://doi.org/10.1371/journal.pone.0154514

Stiremaniii, J. O., Nason, J. D., \& Heard, S. B. (2005). Host-associated genetic differentiation in phytophagous insects: general phenomenon or isolated exceptions? Evidence from a goldenrod-insect community. Evolution , 59: . 2573-2587. https://doi.org/10.1111/j.0014-3820.2005.tb00970.x

Strano, C. P., Malacrino, A., Campolo, O., \& Palmeri, V. (2018). Influence of host plant on Thaumetopoea pityocampa gut bacterial community. Microbial Ecology, 75, 487-494. https://doi.org/10.1007/s00248-017$1019-6$

Tang, X., Freitak, D., Vogel, H., Ping L. Y., Shao Y. Q., Cordero, E. A., Andersen, G., Westermann, M., Heckel, D. G., \& Boland W. (2012). Complexity and variability of gut commensal microbiota in polyphagous lepidopteran larvae. PLoS One, 7, e36978. https://doi.org/10.1371/journal.pone.0036978

Terra, W. R., Dias, R. O., Oliveira, P. L., Ferreira, C., \& Venancio, T. M. (2018). Transcriptomic analyses uncover emerging roles of mucins, lysosome/secretory addressing and detoxification pathways in insect midguts. Current Opinion in Insect Science , 29, 34-40. https://doi.org/10.1016/j.cois.2018.05.015

Trapnell, C., Roberts, A., Goff, L., Pertea, G., Kim, D., Kelley, D. R., Pimentel, H., Salzberg, S. L., Rinn, J. L., \& Pachter, L. (2012). Differential gene and transcript expression analysis of RNA-seq experiments with TopHat and Cufflinks.Nature Protocols , 7, 562-578. https://doi.org/10.1038/nprot.2012.016

van den Bosch, T. J. M., \& Welte, C. U. (2017). Detoxifying symbionts in agriculturally important pest insects. Microbial Biotechnology , 10, 531-540. https://doi.org/10.1111/1751-7915.12483

Vilanova, C., Baixeras, J., Latorre, A., \& Porcar, M. (2016). The generalist inside the specialist: gut bacterial communities of two insect species feeding on toxic plants are dominated byEnterococcus sp. Frontiers in Microbiology , 7, 1005. https://doi.org/10.3389/fmicb.2016.01005

Vogel, H., Musser, R. O., \& Celorio-Mancera, M. D. (2014). Transcriptome responses in herbivorous insects towards host plant and toxin feeding. Annual Plant Reviews: Insect-Plant Interactions , 47, 197-234. https://doi.org/10.1002/9781118829783.ch6

Wei, J. Segraves, K. A., Li, W. Z., Yang, X. K., \& Xue, H. J. (2020). Gut bacterial communities and their contribution to performance of specialist Altica flea beetles. Microbial Ecology , 80, 946-959. https://doi.org/10.1007/s00248-020-01590-x

Weiz, G., Mazzaferro, L. S., Kotik, M., Neher, B. D., Halada, P., Křen, V., \& Breccia, J. D. (2019). The flavonoid degrading fungusAcremonium sp. DSM 24697 produces two diglycosidases with different specificities. Applied Microbiology and Biotechnology , 103, 9493-9504. https://doi.org/10.1007/s00253-019$10180-\mathrm{y}$

Welte, C. U., de Graaf, R. M., van den Bosch, T. J. M., Op den Camp, H.J., van Dam, N. M., \& Jetten, M. S. M. (2016). Plasmids from the gut microbiome of cabbage root fly larvae encode SaxA that catalyzes the conversion of the plant toxin 2-phenylethyl isothiocyanate.Environmental Microbiology , 18, 1379-1390. https://doi.org/10.1111/1462-2920.12997

Whitaker, M. R. L., Salzman, S., Sanders, J., Kaltenpoth, M., \& Pierce, N. E. (2016). Microbial communities of Lycaenid butterflies do not correlate with larval diet.Frontiers in Microbiology , 7, 1920. https://doi.org/10.3389/fmicb.2016.01920 
Wood, D. E., \& Salzberg, S. L. (2014). Kraken: ultrafast metagenomic sequence classification using exact alignments. Genome Biology, 15, R46. https://doi.org/10.1186/gb-2014-15-3-r46

Wybouw, N., Zhurov, V., Martel, C., Bruinsma, K. A., Hendrickx, F., Grbić, V., \& van Leeuwen, T. (2015). Adaptation of a polyphagous herbivore to a novel host plant extensively shapes the transcriptome of herbivore and host. Molecular Ecology , 24, 4647-4663. https://doi.org/10.1111/mec.13330

Xia, X. F., Gurr, G. M.., Vasseur, L., Zheng, D. D., Zhong, H. Z., Qin, B. C., Lin, J. H., Wang, Y., Song, F. Q., Li, Y., Lin, H. L., \& You, M. S. (2017). Metagenomic sequencing of diamondback moth gut microbiome unveils key holobiont adaptations for herbivory. Frontiers in Microbiology , 8, 663. https://doi.org/10.3389/fmicb.2017.00663

Xu, W. D., Lin, H. W., Qiu, F., \& Chen, W. S. (2007). Chemical constituents of Duchesnea indicaFocke. Journal of Shenyang Pharmaceutical University , 24, 402-406.

Xue, H. J., Li, W. Z., \& Yang, X. K. (2009a). Genetic analysis of feeding preference in two related species of Altica (Coleoptera: Chrysomelidae: Alticinae). Ecological Entomology , 34, 74-80. https://doi.org/10.1111/j.1365-2311.2008.01042.x

Xue, H. J., Li, W. Z., Nie, R. E., \& Yang, X. K. (2011). Recent speciation in three closely related sympatric specialists: inferences using multi-locus sequence, post-mating isolation and endosymbiont data.PLoS One, 6, e27834. https://doi.org/10.1371/journal.pone.0027834

Xue, H. J., Magalhães, S., Li, W. Z., \& Yang, X. K. (2009b). Reproductive barriers between two sympatric beetle species specialized on different host plants. Journal of Evolutionary Biology , 22, 2258-2266. https://doi.org/10.1111/j.1420-9101.2009.01841.x

Xue, H. J., Niu, Y. W., Segraves, K. A., Nie, R. E., Hao, Y. J., Zhang, L. L., Cheng, X. C., Zhang, X. W., Li, W. Z., Chen, R. S., \& Yang, X. K. (2021). The draft genome of the specialist flea beetle Altica viridicyanea (Coleoptera: Chrysomelidae). BMC Genomics ,22, 243. https://doi.org/10.1186/s12864-021-07558-6

Xue, H. J., Wei, J. N., Magalhães, S., Zhang, B., Song, K. Q., Liu, J., Li, W. Z., \& Yang, X. K. (2016). Contact pheromones of 2 sympatric beetles are modified by the host plant and affect mate choice.Behavioral Ecology , 27, 895-902. https://doi.org/10.1093/beheco/arv238

Yang, H. L., Wang, J., Lv, Z. Y., Tian, J. X., Peng, Y. D., Peng, X. J., Xu, X., Song, Q. S., Lv, B., Chen, Z. Y., Sun, Z. Y., \& Wang, Z. (2018). Metatranscriptome analysis of the intestinal microorganisms in Pardosa pseudoannulata in response to cadmium stress. Ecotoxicology and Environmental Safety, 159, 1-9. https://doi.org/10.1016/j.ecoenv.2018.04.053

Yang, X. F., Cheng, X. W., Ma, Y. M., \& Kang, Y. X. (2015). Study on chemical compositions of Geranium wilfordii Maxim. (II).Journal of Shaanxi University of Science 83 Technology , 33, 95-98.

Ye, L., \& Yang, J. S. (1996). New ellagic glycosides and known triterpenoids from Duchesnea indicaFocke. Acta Pharmaceutica Sinica, 31, 844-848.

Young, M. D., Wakefield, M. J., Smyth, G. K., \& Oshlack, A. (2010). Gene ontology analysis for RNA-seq: accounting for selection bias.Genome Biology, 11, R14. https://doi.org/10.1186/gb-2010-11-2-r14

Yu, Q. Y., Fang, S. M., Zhang, Z., \& Jiggins, C. D. (2016). The transcriptome response of Heliconius melpomene larvae to a novel host plant. Molecular Ecology , 25, 4850-4865. https://doi.org/10.1111/mec.13826

Yun, J. H., Roh, S. W., Whon, T. W., Jung, M. J., Kim, M. S., Park, D. S., Yoon, C., Nam, Y. D., Kim, Y. J., Choi, J. H., Kim, J. Y., Shin, N. R., Kim, S. H., Lee, W. J., \& Bae, J. W. (2014). Insect gut bacterial diversity determined by environmental habitat, diet, developmental stage, and phylogeny of host. Applied and Environmental Microbiology , 80, 5254-5264. https://doi.org/10.1128/AEM.01226-14

Zhang, S. K., Shu, J. P., Xue, H. J., Zhang, W., Zhang, Y. B., Liu, Y. N., Fang, L. X., Wang, Y. D., \& Wang, H. J. (2020). The gut microbiota in camellia weevils are influenced by plant secondary metabolites and 
contribute to saponin degradation. mSystems , 5, e00692-19. https://doi.org/10.1128/mSystems.00692-19

Zhong, H. Y., Li, F. B., Chen, J. M., Zhang, J. F., \& Li, F. (2017). Comparative transcriptome analysis reveals host-associated differentiation in Chilo suppressalis (Lepidoptera: Crambidae).Scientific Reports , 7, 13778. https://doi.org/10.1038/s41598-017-14137-x

Zhu, F., Moural, T. W., Nelson, D. R., \& Palli, S. R. (2016). A specialist herbivore pest adaptation to xenobiotics through up-regulation of multiple Cytochrome P450s. Scientific Reports , 6, 20421. https://doi.org/10.1038/srep20421

\section{CONFLICT OF INTERESTS}

There are no conflict of interests to declare.

\section{DATA ACCESSIBILITY STATEMENT}

Sequencing data are available at the Genome Sequence Archive in National Genomics Data Center (https://bigd.big.ac.cn/gsa; accession number: XXXXXX).

\section{AUTHOR CONTRIBUTIONS}

Huai-Jun Xue and Xing-Ke Yang conceived the idea; Huai-Jun Xue acquired funding; Jing Wei and Huai-Jun Xue collected data; Jing Wei and Huai-Jun Xue performed analyses; Huai-Jun Xue, Kari Segraves and Jing Wei drafted the manuscript.

Table 1 Sample collection information for the meta-transcriptome analysis. AFDi: Altica fragariae fedDuchesnea indica ; AVGn: A. viridicyanea fed Geranium nepalense ; $\mathrm{F}_{1}$ Di: $\mathrm{F}_{1}$ hybrid fed D. indica ; $\mathrm{F}_{1} \mathrm{Gn}: \mathrm{F}_{1}$ hybrid fed G. nepalense.

\begin{tabular}{llll}
\hline Treatment & Sample number & Plant materials provided & RNA collection date \\
\hline AFDi & T01 & Duchesnea indica & 2017.VIII.10 \\
& T02 & Duchesnea indica & 2017.VIII.10 \\
& T08 & Duchesnea indica & 2017.VIII.31 \\
AVGn & T05 & Geranium nepalens & 2017.IX.26 \\
& T07 & Geranium nepalens & 2017.IX.7 \\
& T09 & Geranium nepalens & 2017.VIII.31 \\
\multirow{5}{*}{ F $_{1}$ Di } & T06 & Duchesnea indica & 2017.IX.26 \\
& T10 & Duchesnea indica & 2017.VIII.24 \\
& T11 & Duchesnea indica & 2017.VIII.24 \\
F $_{1}$ Gn & T03 & Geranium nepalens & 2017.VIII.17 \\
& T04 & Geranium nepalens & 2017.VIII.17 \\
& T12 & Geranium nepalens & 2017.VIII.17 \\
\hline
\end{tabular}

Table 2 Summary of sequence data obtained from Alticaintestinal tissue.

\begin{tabular}{llllll}
\hline Treatment & Sample ID & Raw data & Raw data & Altica & Gut symbionts \\
\hline \multirow{2}{*}{ AFDi } & & Total base (bp) & Number of reads & Number of reads & Number of reads \\
& T01 & $34,982,604,280$ & $234,404,490$ & $37,746,866$ & $55,884,174$ \\
& T02 & $30,708,009,768$ & $205,715,992$ & $39,155,111$ & $58,895,563$ \\
\multirow{2}{*}{ AVGn } & T08 & $31,098,436,954$ & $208,344,922$ & $19,676,553$ & $26,356,925$ \\
& T05 & $30,816,908,400$ & $206,213,834$ & $68,846,937$ & $57,966,230$ \\
& T07 & $30,490,073,786$ & $203,922,072$ & $51,511,204$ & $44,783,777$ \\
\multirow{2}{*}{ F $_{1}$ Di } & T09 & $30,563,115,808$ & $204,576,644$ & $36,293,549$ & $26,630,573$ \\
& T06 & $46,161,460,558$ & $308,512,052$ & $58,327,170$ & $91,461,315$
\end{tabular}




\begin{tabular}{llllll}
\hline Treatment & Sample ID & Raw data & Raw data & Altica & Gut symbionts \\
\hline \multirow{4}{*}{ F $_{1}$ Gn } & T10 & $44,415,301,278$ & $298,011,082$ & $27,186,052$ & $22,127,621$ \\
& T11 & $39,567,947,548$ & $265,037,190$ & $37,318,556$ & $38,344,031$ \\
& T03 & $34,493,392,948$ & $230,705,486$ & $72,218,420$ & $75,605,464$ \\
\multirow{2}{*}{ mean } & T04 & $30,530,791,558$ & $204,207,512$ & $64,598,253$ & $69,481,168$ \\
& T12 & $32,134,024,410$ & $214,920,354$ & $63,690,321$ & $66,853,217$ \\
& & $34,663,505,608$ & $232,047,636$ & $48,047,416$ & $52,865,838$ \\
\hline
\end{tabular}

Table 3 Summary of gene annotation obtained from Alticaintestinal tissue.

\begin{tabular}{ll}
\hline Annotated databases & Gene number \\
\hline COG & 3,224 \\
GO & 2,005 \\
KEGG & 3,244 \\
KOG & 6,793 \\
Pfam & 7,495 \\
Swiss-Prot & 3,516 \\
eggNOG & 8,722 \\
nr & 6,110 \\
All & 8,843 \\
\hline
\end{tabular}

Table 4 Number of differentially expressed genes (DEGs) identified in Altica. Annotations are broken down by database. AFDi: Altica fragariae fed Duchesnea indica; AVGn:A. viridicyanea fed Geranium nepalense ; $\mathrm{F}_{1}$ Di: $\mathrm{F}_{1}$ hybrid fed D. indica $; \mathrm{F}_{1} \mathrm{Gn}$ : $\mathrm{F}_{1}$ hybrid fed G. nepalense ; DiT: AFDi $+\mathrm{F}_{1} \mathrm{Di}$; GnT: AVGn + $\mathrm{F}_{1} \mathrm{Gn}$.

\begin{tabular}{lllllllll}
\hline DEG Set & GO & KEGG & KOG & NR & Pfam & Swiss-Prot & eggNOG & Total \\
\hline AFDi vs AVGn & 744 & 686 & 1,047 & 1,505 & 1,232 & 938 & 1,440 & 1512 \\
DiT vs GnT & 102 & 94 & 167 & 228 & 176 & 139 & 218 & 230 \\
F $_{1}$ Di vs F $_{1}$ Gn & 65 & 66 & 109 & 149 & 124 & 88 & 146 & 154 \\
\hline
\end{tabular}

Table 5 Enrichment of Altica gut DEGs based on the KEGG database (20 most credible pathways). Bold indicates DEGs related to detoxification and digestion.

\begin{tabular}{llll}
\hline & AFDi vs. AVGn & $\mathrm{F}_{1}$ Di vs. $\mathrm{F}_{1} \mathrm{Gn}$ & DiT vs. GnT \\
\hline 1 & Glutathione metabolism & Arachidonic acid metabolism & Glutathione metabolis \\
2 & Cysteine and methionine metabolism & Cyanoamino acid metabolism & Cyanoamino acid metabol \\
3 & Glycine, serine and threonine metabolism & Taurine and hypotaurine metabolism & Taurine and hypotaurine \\
4 & Lysosome & Lysosome & Arachidonic acid metabol \\
5 & Arginine and proline metabolism & Glutathione metabolism & Lysosome \\
6 & Selenocompound metabolism & Proteoglycans & Glycosaminoglycan degrac \\
7 & Biosynthesis of amino acids & N-Glycan biosynthesis & Pyruvate metabolism \\
8 & Steroid biosynthesis & Other glycan degradation & Lysine degradation \\
9 & Fatty acid degradation & ECM-receptor interaction & Histidine metabolism \\
10 & $\beta$-Alanine metabolism & Lysine biosynthesis & Cysteine and methionine \\
11 & Vitamin B6 metabolism & Glycosaminoglycan degradation & Biosynthesis of amino aci
\end{tabular}




\begin{tabular}{llll}
\hline & AFDi vs. AVGn & $\mathrm{F}_{1}$ Di vs. $\mathrm{F}_{1} \mathrm{Gn}$ & DiT vs. GnT \\
\hline 12 & Glycosaminoglycan degradation & Pyruvate metabolism & ABC transporters \\
13 & Glycosphingolipid biosynthesis - ganglio series & Drug metabolism - other enzymes & Arginine and proline meta \\
14 & N-Glycan biosynthesis & Lysine degradation & Other glycan degradation \\
15 & Tryptophan metabolism & $a$-Linolenic acid metabolism & Glycine, serine and threor \\
16 & Pyruvate metabolism & Pentose and glucuronate interconversions & Pentose and glucuronate \\
17 & Histidine metabolism & 2-Oxocarboxylic acid metabolism & Tryptophan metabolis \\
18 & Carbon metabolism & Starch and sucrose metabolism & $\beta$-Alanine metabolism \\
19 & Alanine, aspartate and glutamate metabolism & Fructose and mannose metabolism & Drug metabolism - cyt \\
20 & Drug metabolism - cytochrome P450 & ABC transporters & Ascorbate and aldarate m \\
\hline
\end{tabular}

Table 6 Comparison of microbial diversity per sample. AFDi:Altica fragariae, AVGn: A. viridicyanea, $\mathrm{F}_{1} \mathrm{Di}$ : $\mathrm{F}_{1}$ hybrid fed Duchesnea indica, $\mathrm{F}_{1} \mathrm{Gn}$ : $\mathrm{F}_{1}$ hybrid fed

Geranium nepalense.

\begin{tabular}{llllll}
\hline & & Percentage & Percentage & Percentage & Percentage \\
\hline Treatment & Sample ID & Archaea & Bacteria & Fungi & Viruses \\
AFDi & T01 & 1.81 & 59.87 & 33.90 & 4.44 \\
& T02 & 1.99 & 58.53 & 35.36 & 4.12 \\
& T08 & 1.64 & 63.07 & 29.98 & 5.31 \\
& mean & 1.81 & 60.49 & 33.08 & 4.63 \\
AVGn & T05 & 2.07 & 55.38 & 38.08 & 4.47 \\
& T07 & 1.90 & 54.48 & 40.22 & 3.40 \\
& T09 & 1.74 & 56.90 & 37.01 & 4.35 \\
& mean & 1.91 & 55.58 & 38.44 & 4.07 \\
F $_{1}$ Di & T06 & 1.96 & 59.72 & 33.65 & 4.68 \\
& T10 & 1.73 & 65.27 & 29.74 & 3.25 \\
& T11 & 1.63 & 64.50 & 30.40 & 3.48 \\
& Fean & 1.77 & 63.16 & 31.26 & 3.80 \\
F $_{1}$ Gn & T03 & 2.37 & 57.23 & 37.05 & 3.35 \\
& T04 & 2.19 & 57.66 & 36.70 & 3.44 \\
& T12 & 1.92 & 58.48 & 35.79 & 3.80 \\
Mean & mean & 2.16 & 57.79 & 36.51 & 3.53 \\
\hline
\end{tabular}

Table 7. Number of microbial taxa identified in the guts of Altica species. AFDi: Altica fragariae, AVGn: A. viridicyanea, $\mathrm{F}_{1}$ Di: $\mathrm{F}_{1}$ hybrid fedDuchesnea indica, $\mathrm{F}_{1} \mathrm{Gn}$ : $\mathrm{F}_{1}$ hybrid fed Geranium nepalense.

\begin{tabular}{lllllllllllll}
\hline & & AFDi & AFDi & AFDi & AVGn & AVGn & AVGn & $\mathbf{F}_{\mathbf{1}}$ Di & $\mathbf{F}_{\mathbf{1}}$ Di & $\mathbf{F}_{\mathbf{1}}$ Di & $\mathbf{F}_{\mathbf{1}} \mathbf{G n}$ & $\mathbf{F}_{\mathbf{1}} \mathbf{C}$ \\
\hline \multirow{3}{*}{ Phylum level } & & T01 & T02 & T08 & T05 & T07 & T09 & T06 & T10 & T11 & T03 & T04 \\
& Archaea & 11 & 10 & 10 & 10 & 10 & 11 & 10 & 10 & 10 & 11 & 11 \\
& Bacteria & 98 & 92 & 84 & 95 & 95 & 92 & 82 & 80 & 93 & 90 & 91 \\
& Fungi & 8 & 7 & 7 & 8 & 8 & 8 & 8 & 7 & 8 & 7 & 8 \\
& Viruses & 1 & 1 & 1 & 1 & 1 & 1 & 1 & 1 & 1 & 1 & 1 \\
Class level & Total & 118 & 110 & 102 & 114 & 114 & 112 & 101 & 98 & 112 & 109 & 111 \\
& Archaea & 6 & 6 & 6 & 8 & 8 & 9 & 6 & 6 & 6 & 8 & 9 \\
& Bacteria & 79 & 74 & 74 & 77 & 77 & 74 & 72 & 72 & 75 & 75 & 75 \\
& Fungi & 39 & 39 & 38 & 40 & 40 & 41 & 39 & 38 & 39 & 39 & 40
\end{tabular}




\begin{tabular}{|c|c|c|c|c|c|c|c|c|c|c|c|c|}
\hline & & AFDi & AFDi & AFDi & AVGn & AVGn & AVGn & $\mathrm{F}_{1} \mathrm{Di}$ & $\mathrm{F}_{1} \mathrm{Di}$ & $\mathrm{F}_{1} \mathrm{Di}$ & $\mathrm{F}_{1} \mathrm{Gn}$ & $F_{1}$ \\
\hline & Viruses & 4 & 3 & 4 & 3 & 3 & 3 & 3 & 4 & 4 & 3 & 3 \\
\hline & Total & 128 & 122 & 122 & 128 & 128 & 127 & 120 & 120 & 124 & 125 & 127 \\
\hline \multirow[t]{5}{*}{ Order level } & Archaea & 19 & 19 & 18 & 20 & 20 & 21 & 18 & 18 & 19 & 19 & 20 \\
\hline & Bacteria & 154 & 145 & 141 & 157 & 154 & 150 & 140 & 141 & 147 & 153 & 149 \\
\hline & Fungi & 97 & 95 & 92 & 97 & 97 & 97 & 93 & 92 & 96 & 95 & 95 \\
\hline & Viruses & 8 & 7 & 9 & 9 & 9 & 9 & 10 & 10 & 10 & 9 & 9 \\
\hline & Total & 278 & 266 & 260 & 283 & 280 & 277 & 261 & 261 & 272 & 276 & 273 \\
\hline \multirow[t]{5}{*}{ Family level } & Archaea & 22 & 21 & 20 & 22 & 23 & 23 & 20 & 19 & 21 & 21 & 22 \\
\hline & Bacteria & 352 & 331 & 317 & 357 & 349 & 345 & 321 & 309 & 330 & 347 & 342 \\
\hline & Fungi & 218 & 216 & 209 & 217 & 218 & 215 & 208 & 206 & 215 & 211 & 212 \\
\hline & Viruses & 37 & 35 & 39 & 45 & 38 & 45 & 45 & 41 & 42 & 38 & 38 \\
\hline & Total & 629 & 603 & 585 & 641 & 628 & 628 & 594 & 575 & 608 & 617 & 614 \\
\hline \multirow[t]{5}{*}{ Genus level } & Archaea & 39 & 36 & 30 & 40 & 42 & 39 & 33 & 31 & 34 & 37 & 37 \\
\hline & Bacteria & 1063 & 973 & 865 & 1108 & 1080 & 1048 & 922 & 824 & 956 & 1084 & \\
\hline & Fungi & 394 & 391 & 380 & 398 & 399 & 392 & 378 & 372 & 390 & 385 & 386 \\
\hline & Viruses & 69 & 68 & 70 & 78 & 69 & 78 & 74 & 71 & 73 & 71 & 71 \\
\hline & Total & 1565 & 1468 & 1345 & 1624 & 1590 & 1557 & 1407 & 1298 & 1453 & 1577 & \\
\hline \multirow[t]{5}{*}{ Species level } & Archaea & 129 & 115 & 90 & 126 & 127 & 121 & 98 & 92 & 109 & 116 & 11 \\
\hline & Bacteria & 3072 & 2653 & 2322 & 3330 & 3211 & 3140 & 2491 & 2097 & 2628 & 3252 & \\
\hline & Fungi & 765 & 761 & 726 & 776 & 774 & 759 & 721 & 701 & 749 & 751 & 75 \\
\hline & Viruses & 251 & 250 & 269 & 278 & 267 & 288 & 277 & 254 & 271 & 265 & \\
\hline & Total & 4218 & 3780 & 3408 & 4511 & 4380 & 4309 & 3588 & 3145 & 3758 & 4385 & \\
\hline
\end{tabular}

Table 8 Statistical results for alpha -diversity of gut microbial communities. 'Pooled' refers to tests of the pooled data (Archaea, Bacteria, Fungi, Virus), '4 groups' refers to comparisons across the beetle species and feeding treatments (AFDi, AVGn, $\mathrm{F}_{1} \mathrm{Di}, \mathrm{F}_{1} \mathrm{Gn}$ ), and '2 groups' are comparisons of diversity indices between beetles fed Duchesnea indica (AFDi $\left.+\mathrm{F}_{1} \mathrm{Di}\right)$ and those fed Geranium nepalense ( $\left.\mathrm{AVGn}+\mathrm{F}_{1} \mathrm{Gn}\right)$. Nonparametric Mann-Whitney (for two groups) or Kruskal-Wallis (for four groups) tests were used to assess whether there were differences among treatments for each estimators.

\begin{tabular}{lllll}
\hline Treatment & P value & P value & P value & P value \\
\hline & ACE & Chao1 & Shannon & Simpson \\
Pooled / 4 groups & 0.024 & 0.024 & 0.154 & 0.036 \\
Pooled / 2 groups & 0.002 & 0.002 & 0.485 & 0.699 \\
Bacteria / 4 groups & 0.022 & 0.022 & 0.084 & 0.027 \\
Bacteria / 2 groups & 0.002 & 0.002 & 0.699 & 0.132 \\
Fungus / 4 groups & 0.147 & 0.113 & 0.536 & 0.086 \\
Fungus / 2 groups & 0.818 & 0.937 & 1.000 & 0.132 \\
\hline
\end{tabular}

Table 9 Statistical results for beta -diversity of gut microbial communities. 'Pooled' refers to tests of the pooled data (Archaea, Bacteria, Fungi, Virus), '4 groups' refers to comparisons across the beetle species and feeding treatments (AFDi, AVGn, $\mathrm{F}_{1} \mathrm{Di}, \mathrm{F}_{1} \mathrm{Gn}$ ), and '2 groups' are comparisons of diversity indices between beetles fed Duchesnea indica (AFDi, $\mathrm{F}_{1} \mathrm{Di}$ ) and those fed Geranium nepalense (AVGn, $\mathrm{F}_{1} \mathrm{Gn}$ ).

\begin{tabular}{lllll}
\hline Treantment & Distance method & Statistical method & Statistics & p-value \\
\hline Pooled, 4 groups & Bray-Curtis Index & PerMANOVA & F-value: 8.250; R-squared: 0.756 & $<0.001$ \\
& & ANOSIM & R: 0.830 & $<0.001$
\end{tabular}




\begin{tabular}{|c|c|c|c|c|}
\hline Treantment & Distance method & Statistical method & Statistics & p-value \\
\hline & Jaccard & PerMANOVA & F-value: $6.983 ; \mathrm{R}$-squared: 0.724 & $<0.001$ \\
\hline & & ANOSIM & $\mathrm{R}: 0.830$ & $<0.001$ \\
\hline \multirow[t]{4}{*}{ Pooled, 2 groups } & Bray-Curtis Index & PerMANOVA & F-value: $6.321 ; \mathrm{R}$-squared: 0.387 & $<0.002$ \\
\hline & & ANOSIM & $\mathrm{R}: 0.489$ & $<0.002$ \\
\hline & Jaccard & PerMANOVA & F-value: 5.378; R-squared: 0.350 & $<0.002$ \\
\hline & & ANOSIM & R: 0.489 & $<0.002$ \\
\hline \multirow[t]{4}{*}{ Bacteria, 4 groups } & Bray-Curtis Index & PerMANOVA & F-value: $10.572 ; \mathrm{R}$-squared: 0.799 & $<0.001$ \\
\hline & & ANOSIM & R: 0.929 & $<0.001$ \\
\hline & Jaccard & PerMANOVA & F-value: 8.836 ; R-squared: 0.768 & $<0.001$ \\
\hline & & ANOSIM & R: 0.929 & $<0.001$ \\
\hline \multirow[t]{4}{*}{ Bacteria, 2 groups } & Bray-Curtis Index & PerMANOVA & F-value: $6.942 ;$ R-squared: 0.410 & $<0.002$ \\
\hline & & ANOSIM & $\mathrm{R}: 0.556$ & $<0.002$ \\
\hline & Jaccard & PerMANOVA & F-value: $5.957 ; \mathrm{R}$-squared: 0.373 & $<0.002$ \\
\hline & & ANOSIM & $\mathrm{R}: 0.556$ & $<0.002$ \\
\hline \multirow[t]{4}{*}{ Fungus, 4 groups } & Bray-Curtis Index & PerMANOVA & F-value: $10.538 ; \mathrm{R}$-squared: 0.798 & $<0.001$ \\
\hline & & ANOSIM & R: 0.932 & $<0.001$ \\
\hline & Jaccard & PerMANOVA & F-value: $8.808 ;$ R-squared: 0.768 & $<0.001$ \\
\hline & & ANOSIM & $\mathrm{R}: 0.932$ & $<0.001$ \\
\hline \multirow[t]{4}{*}{ Fungus, 2 groups } & Bray-Curtis Index & PerMANOVA & F-value: $7.043 ;$ R-squared: 0.413 & $<0.002$ \\
\hline & & ANOSIM & $\mathrm{R}: 0.489$ & $<0.002$ \\
\hline & Jaccard & PerMANOVA & F-value: $6.132 ; \mathrm{R}$-squared: 0.380 & $<0.002$ \\
\hline & & ANOSIM & $\mathrm{R}: 0.489$ & $<0.002$ \\
\hline
\end{tabular}

Table 10. The role of beetle host ( $\mathrm{AF}, \mathrm{AV}$ and $\mathrm{F}_{1}$ ) and host diet (Duchesnea indica and Geranium nepalense ) in structuring gut microbial communities within Altica species. The analyses were carried out using twoway PerMANOVAs with "beetle species" and "plant diet" as main effects. 'Pooled' refers to tests of the pooled microbial data (Archaea, Bacteria, Fungi, Virus).

\begin{tabular}{llllllll}
\hline Microbal data & Beetle host & Beetle host & Beetle host & Beetle host & Beetle host & Host diet & Host diet \\
\hline & Jaccard & Jaccard & Bray-Curtis & Bray-Curtis & Bray-Curtis & Jaccard & Jaccard \\
& $F$ & $p$ & $p$ & $F$ & $p$ & $F$ & $p$ \\
Pooled & 1.872 & $\mathrm{P}=0.001$ & $\mathrm{P}=0.001$ & 5.515 & $<0.001$ & 4.000 & $<0.001$ \\
Bacteria & 1.749 & $\mathrm{P}=0.002$ & $\mathrm{P}=0.002$ & 6.844 & $<0.001$ & 3.850 & $<0.001$ \\
Fungus & 1.850 & $\mathrm{P}=0.003$ & $\mathrm{P}=0.003$ & 6.250 & $<0.001$ & 3.522 & $<0.001$ \\
\hline
\end{tabular}

Table 11. Number of gut microbes that showed differential relative abundance in the comparisons estimated using DESeq2. AFDi:Altica fragariae, AVGn: A. viridicyanea, $\mathrm{F}_{1} \mathrm{Di}_{\mathrm{i}} \mathrm{F}_{1}$ hybrid fed Duchesnea indica, $\mathrm{F}_{1} \mathrm{Gn}$ : $\mathrm{F}_{1}$ hybrid fedGeranium nepalense, DiT: AFDi and $\mathrm{F}_{1}$ Di, GnT: AVGn and $\mathrm{F}_{1} \mathrm{Gn}$. 'Pooled' refers to tests of the pooled microbial data (Archaea, Bacteria, Fungi and Virus).

\begin{tabular}{llll}
\hline Comparison & Data & Genus level & Species level \\
\hline AFDi vs AVGn & Pooled & 546 & 1489 \\
& Bacteria & 423 & 1335 \\
& Fungi & 112 & 213 \\
$\mathrm{~F}_{1}$ Di vs $\mathrm{F}_{1}$ Gn & Pooled & 257 & 502 \\
& Bacteria & 152 & 348 \\
& Fungi & 84 & 134
\end{tabular}




\begin{tabular}{llll}
\hline Comparison & Data & Genus level & Species level \\
\hline DiT vs GnT & Pooled & 336 & 961 \\
& Bacteria & 282 & 937 \\
& Fungi & 63 & 104 \\
\hline
\end{tabular}

Table 12 Predicted genes identified in the Altica gut microbiota.

\begin{tabular}{llll}
\hline Treatment & Sample ID & Gene number & Total length $(\mathbf{b p})$ \\
\hline AFDi & T01 & 127,949 & $24,661,584$ \\
& T02 & 125,938 & $26,756,012$ \\
\multirow{2}{*}{ AVGn } & T08 & 66,123 & $11,942,469$ \\
& T05 & 135,026 & $27,882,530$ \\
& T07 & 119,768 & $24,252,314$ \\
$\mathbf{F}_{\mathbf{1}} \mathbf{D i}$ & T09 & 88,259 & $15,206,960$ \\
& T06 & 145,987 & $25,427,366$ \\
& T10 & 65,305 & $11,516,232$ \\
$\mathbf{F}_{\mathbf{1}} \mathbf{G n}$ & T11 & 106,638 & $18,312,537$ \\
& T03 & 155,268 & $27,487,677$ \\
& T04 & 139,707 & $25,107,670$ \\
& T12 & 135,778 & $24,394,360$ \\
\hline
\end{tabular}

Table 13 Results of functional gene annotation of Alticagut microbes.

\begin{tabular}{lllll}
\hline Treatment & Sample ID & KEGG & KEGG & eggNOG \\
\hline \multirow{4}{*}{ AFDi } & & KEGG Orthology (annotated to KO) & Number of annotated pathways & NOG \\
& T01 & 3,483 & 150 & 9,979 \\
& T02 & 3,216 & 147 & 9,366 \\
\multirow{3}{*}{ AVGn } & T08 & 3,551 & 148 & 8,992 \\
& T05 & 3,695 & 156 & 11,901 \\
& T07 & 3,847 & 156 & 11,831 \\
\multirow{2}{*}{$F_{1}$ Di } & T09 & 3,612 & 156 & 11,367 \\
& T06 & 3,177 & 148 & 8,846 \\
& T10 & 3,002 & 146 & 8,392 \\
\multirow{2}{*}{$F_{1}$ Gn } & T11 & 3,250 & 149 & 9,233 \\
& T03 & 4,484 & 158 & 12,093 \\
& T04 & 3,695 & 157 & 11,391 \\
& T12 & 3,681 & 156 & 10,727 \\
\hline
\end{tabular}

Table 14 Beta diversity of microbial community function. AFDi: Altica fragariae, AVGn: A. viridicyanea, $\mathrm{F}_{1}$ Di: $\mathrm{F}_{1}$ hybrid fed Duchesnea indica, $\mathrm{F}_{1} \mathrm{Gn}$ : $\mathrm{F}_{1}$ hybrid fedGeranium nepalense, DiT: AFDi + $\mathrm{F}_{1} \mathrm{Di}, \mathrm{GnT}: \mathrm{AVGn}+\mathrm{F}_{1} \mathrm{Gn}$.

\begin{tabular}{lllllllll}
\hline & Statistical method & Anosim & Anosim & Anosim & Anosim & Permanova & Permanova & Permanova \\
\hline \multirow{3}{*}{ Database } & Distance method & Jaccard & Jaccard & Bray-curtis & Bray-curtis & Jaccard & Jaccard & Bray-curtis \\
KO & Comparison & R & P & R & P & R & P & R \\
& AFDi-AVGn & 0.444 & 0.100 & 1.000 & 0.100 & 0.368 & 0.100 & 0.882
\end{tabular}




\begin{tabular}{|c|c|c|c|c|c|c|c|c|}
\hline & Statistical method & Anosim & Anosim & Anosim & Anosim & Permanova & Permanova & Permanova \\
\hline \multirow{7}{*}{ NOG } & $\mathrm{F}_{1} \mathrm{Di}-\mathrm{F}_{1} \mathrm{Gn}$ & 0.778 & 0.100 & 0.778 & 0.100 & 0.549 & 0.100 & 0.729 \\
\hline & DiT-GnT & 0.637 & 0.003 & 0.824 & 0.002 & 0.350 & 0.001 & 0.550 \\
\hline & $\mathrm{AF}-\mathrm{AV}-\mathrm{F}_{1} \mathrm{Di}-\mathrm{F}_{1} \mathrm{Gn}$ & 0.534 & 0.001 & 0.904 & 0.001 & 0.525 & 0.001 & 0.800 \\
\hline & AFDi-AVGn & 1.000 & 0.100 & 1.000 & 0.100 & 0.714 & 0.001 & 0.749 \\
\hline & $\mathrm{F}_{1} \mathrm{Di}_{\mathrm{i}} \mathrm{F}_{1} \mathrm{Gn}$ & 1.000 & 0.100 & 0.778 & 0.100 & 0.660 & 0.001 & 0.729 \\
\hline & DiT-GnT & 1.000 & 0.003 & 0.817 & 0.003 & 0.578 & 0.001 & 0.516 \\
\hline & $\mathrm{AF}-\mathrm{AV}-\mathrm{F}_{1} \mathrm{Di}-\mathrm{F}_{1} \mathrm{Gn}$ & 0.809 & 0.001 & 0.867 & 0.001 & 0.716 & 0.001 & 0.765 \\
\hline
\end{tabular}

Table 15. Differentially expressed genes (DEGs) of intestinal microorganisms identified in beetles fed different plants. AFDi:Altica fragariae, AVGn: A. viridicyanea, $\mathrm{F}_{1} \mathrm{Di}$ : $\mathrm{F}_{1}$ hybrid fed Duchesnea indica, $\mathrm{F}_{1} \mathrm{Gn}$ : $\mathrm{F}_{1}$ hybrid fedGeranium nepalense, DiT: AFDi $+\mathrm{F}_{1}$ Di, GnT: AVGn $+\mathrm{F}_{1} \mathrm{Gn}$.

\begin{tabular}{lllll}
\hline Comparison & & Up-regulated & Down-regulated & Total \\
\hline AFDi vs. AVGn & All genes & 25,944 & 20,711 & 46,655 \\
F $_{1}$ Di vs. F ${ }_{1}$ Gn & & 1,148 & 568 & 1,716 \\
DiT vs. GnT & 5,751 & 1,847 & 7,598 \\
AFDi vs. AVGn & KEGG & 2,319 & 1154 & 3,473 \\
F $_{1}$ Di vs. F F 19 & 342 & 94 & 436 \\
DiT vs. GnT & 1,020 & 184 & 1,204 \\
\hline
\end{tabular}

Table 16 The top KEGG terms annotated by DEGs in the intestinal microorganisms of Altica species fed different plant species. AFDi: Altica fragariae fed Duchesnea indica, AVGn:A. viridicyanea fed Geranium nepalense, $\mathrm{F}_{1}$ Di: $\mathrm{F}_{1}$ hybrid fed Duchesnea indica, $\mathrm{F}_{1} \mathrm{Gn}$ : $\mathrm{F}_{1}$ hybrid fedGeranium nepalense, DiT: AFDi + $\mathrm{F}_{1} \mathrm{Di}$, GnT: AVGn $+\mathrm{F}_{1} \mathrm{Gn}$.

\begin{tabular}{lllll}
\hline AFDi vs. AVGn & & & F $_{\mathbf{1}}$ Di vs. $\mathbf{F}_{\mathbf{1}} \mathbf{G n}$ \\
\hline & Kegg pathway & Corrected P-value & Enrichment factor & Kegg pathway \\
$\mathbf{1}$ & ko00460 Cyanoamino acid metabolism & $<0.001$ & 2.39 & ko03010 Ribosome \\
$\mathbf{2}$ & ko00500 Starch and sucrose metabolism & $<0.001$ & 2.16 & ko00630 Glyoxylate \\
$\mathbf{3}$ & ko00040 Pentose and glucuronate interconversions & $<0.001$ & 2.19 & ko01200 Carbon me \\
$\mathbf{4}$ & ko00590 Arachidonic acid metabolism & $<0.001$ & 3.55 & ko00350 Tyrosine m \\
$\mathbf{5}$ & ko00480 Glutathione metabolism & $<0.001$ & 1.76 & \\
$\mathbf{6}$ & ko04011 MAPK signaling pathway - yeast & 0.007 & 1.59 & \\
$\mathbf{7}$ & ko00511 Other glycan degradation & 0.021 & 2.18 & \\
$\mathbf{8}$ & & & \\
\hline
\end{tabular}

\section{Figure Legends}

Figure 1 Heatmap of Altica gut gene expression comparison between samples based on Pearson's Correlation Coefficient r (AFDi: T01, T02, T08; AVGn: T05, T07, T09; F 1 Di: T06, T10, T11; F1 Gn: T03, T04, T12). Different colors on the heatmap show the correlation coefficients.

Figure 2 Enriched differentially expressed genes in KEGG pathways in three comparisions: a, AFDi vs. AVGn; b, DiT vs. GnT; c, $\mathrm{F}_{1}$ Di vs. $\mathrm{F}_{1}$ Gn. AFDi: Altica fragariae, AVGn: A. viridicyanea, $\mathrm{F}_{1}$ Di: $\mathrm{F}_{1}$ hybrid fed Duchesnea indica, $\mathrm{F}_{1} \mathrm{Gn}$ : $\mathrm{F}_{1}$ hybrid fed Geranium nepalense, DiT: AFDi $+\mathrm{F}_{1} \mathrm{Di}$, GnT: AVGn $+\mathrm{F}_{1} \mathrm{Gn}$. 
Figure 3 Principal coordinates analysis (PCoA) of gut microbial communities in four beetle treatments using the Bray-Curtis (a) and Jaccard (b) distance methods and based on the pooled microbial data (Archaea, Bacteria, Fungi and Virus).

Figure 4 Principal Components Analysis (PCA) of functional genes from the gut microbial communities based on the KEGG Orthology (KO) dataset.

Figure S1 Enriched GO terms identified in Altica. AFDi:Altica fragariae, AVGn: A. viridicyanea, $\mathrm{F}_{1} \mathrm{Di}$ : $\mathrm{F}_{1}$ hybrid fed Duchesnea indica, $\mathrm{F}_{1} \mathrm{Gn}$ : $\mathrm{F}_{1}$ hybrid fedGeranium nepalense, DiT: AFDi $+\mathrm{F}_{1} \mathrm{Di}$, GnT: $\mathrm{AVGn}+\mathrm{F}_{1} \mathrm{Gn}$.

Figure S2Differentially expressed genes (DEGs) of beetles. AFDi: Altica fragariae, AVGn:A. viridicyanea, $\mathrm{F}_{1}$ Di: $\mathrm{F}_{1}$ hybrid fed Duchesnea indica , $\mathrm{F}_{1} \mathrm{Gn}$ : $\mathrm{F}_{1}$ hybrid fed Geranium nepalense, DiT: AFDi + $\mathrm{F}_{1} \mathrm{Di}$, GnT: AVGn $+\mathrm{F}_{1} \mathrm{Gn}$.

Table S1 List of the detoxification-related genes inAltica annotated in the present study.

Table S2 List of the detoxification-related differentially expressed genes of Altica species. AFDi: Altica fragariae, AVGn: A. viridicyanea, $\mathrm{F}_{1}$ Di: $\mathrm{F}_{1}$ hybrid fed Duchesnea indica, $\mathrm{F}_{1} \mathrm{Gn}$ : $\mathrm{F}_{1}$ hybrid fed Geranium nepalense, DiT: AFDi $+\mathrm{F}_{1} \mathrm{Di}$, GnT: AVGn $+\mathrm{F}_{1} \mathrm{Gn}$.

Table S3 Relative abundance of gut microorganisms at phylum level in each sample.

Table S4 Relative abundance of gut microoganisms at genus level in each sample.

Table S5 Relative abundance of gut microoganisms at species level in each sample.

Table S6 Microbial composition across three comparisons. AFDi:Altica fragariae fed Duchesnea indica, AVGn: A. viridicyanea fed Geranium nepalense, $\mathrm{F}_{1}$ Di: $\mathrm{F}_{1}$ hybrid fed Duchesnea indica, $\mathrm{F}_{1} \mathrm{Gn}$ : $\mathrm{F}_{1}$ hybrid fed Geranium nepalense, DiT: AFDi $+\mathrm{F}_{1} \mathrm{Di}$, GnT: AVGn $+\mathrm{F}_{1} \mathrm{Gn}$.

Table S7 Differentially expressed genes identified to KEGG pathways. AFDi: Altica fragariae fed Duchesnea indica, AVGn: A. viridicyanea fed Geranium nepalense, $\mathrm{F}_{1} \mathrm{Di}_{1} \mathrm{~F}_{1}$ hybrid fedDuchesnea indica, $\mathrm{F}_{1}$ Gn: $\mathrm{F}_{1}$ hybrid fed Geranium nepalense, DiT: AFDi $+\mathrm{F}_{1} \mathrm{Di}$, GnT: AVGn $+\mathrm{F}_{1} \mathrm{Gn}$.

\section{Hosted file}

Fig 1.eps available at https://authorea.com/users/438097/articles/539365-parallel-metatranscriptome-analysis-reveals-degradation-of-plant-secondary-metabolites-by-beetlesand-their-gut-symbionts

\section{Hosted file}

Fig 2.eps available at https://authorea.com/users/438097/articles/539365-parallel-metatranscriptome-analysis-reveals-degradation-of-plant-secondary-metabolites-by-beetlesand-their-gut-symbionts

\section{Hosted file}

Fig 3.eps available at https://authorea.com/users/438097/articles/539365-parallel-metatranscriptome-analysis-reveals-degradation-of-plant-secondary-metabolites-by-beetlesand-their-gut-symbionts 


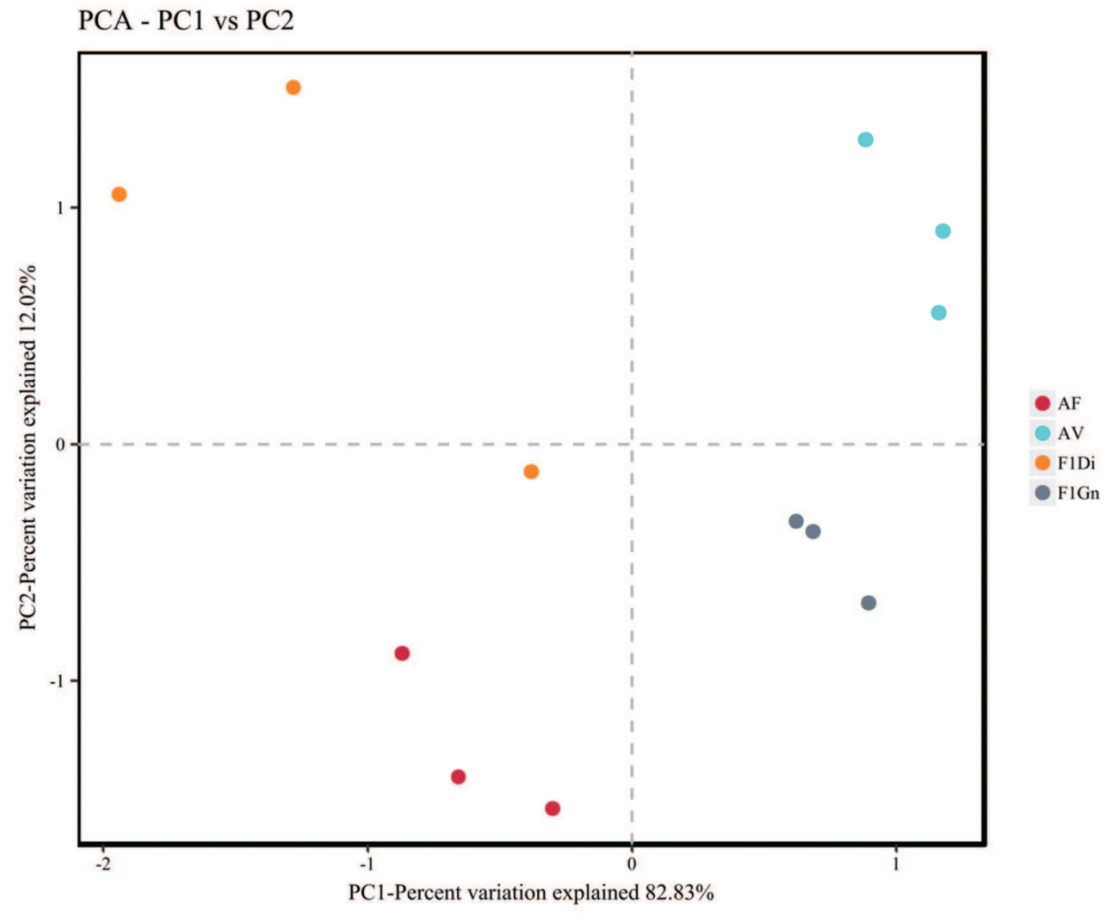

